\title{
Wind-driven barotropic gyre II: Effects of eddies and low interior viscosity
}

\author{
by Baylor Fox-Kemper ${ }^{1,2}$
}

\begin{abstract}
Using boundary-enhanced viscosity to control the mean circulation, a simple model can be created and used for study of strong inertial effects in a western-intensified calculation. The simplicity allows for a greater number of strongly-inertial numerical experiments than computationally feasible in a general circulation model.

This paper is an introduction to the behavior of this model, covering its general features. Some of the inertial phenomena, including the primary balances of the boundary current and basin interior, the temporal behavior, and the changes in the mean state across parameter space are presented. The analysis of these phenomena focuses on the effects of eddies and the type of eddies present. The low interior viscosity allows for more pronounced eddy effects.

As this model is intended for use in future studies, many of the diagnostic tools found to be useful here are likely to be reused effectively.
\end{abstract}

\section{Introduction}

Fox-Kemper and Pedlosky (2004) demonstrate that the circulation strength of the single-gyre can be controlled with lower interior viscosity by a combination of eddy vorticity transport and boundary-enhanced viscosity. Once this control is achieved, it is possible to produce calculations which remain western intensified at a much higher Reynolds number in the basin interior than is possible with constant viscosity.

Changing the viscosity in an inertial model is significantly more interesting than changing the viscosity in a linear Munk model. In the latter case, the widths of boundary currents change, but the character of the solution does not. In an inertial model, the results of changing the viscosity may be qualitative as well as quantitative. This paper explores some of the behavior of the strongly inertial, western-intensified Munk model, and presents the most surprising and stimulating results found during this investigation. It is demonstrated that the boundary layer vorticity balance, the basin interior vorticity balance, and the temporal character are all qualitatively affected by lowering the interior viscosity. A

1. MIT/WHOI Joint Program in Oceanography and Ocean Engineering, Woods Hole, Massachusetts, 02543, U.S.A.

2. Present address: Program in Atmospheric and Oceanic Sciences, Princeton University, Princeton, New Jersey, 08544, U.S.A.email: baylor@princeton.edu 
particularly nonlinear effect is also discussed: the existence of solutions with substantially different viscosities but the same time-mean flow. The simplicity of the Fox-Kemper and Pedlosky (2004) model allows for significant exploration of parameter space at fine resolution.

A recurring theme in the analysis of the model results is that ensuring western intensification seems to ensure a weakly nonlinear and thus nearly Sverdrup (1947) interior. On the other hand, with low interior viscosity, the dynamics of the boundary current must be dominantly nonlinear, requiring eddies and inertia in the primary boundary-layer balance. The strongly heterogeneous character of this model is typical of the ocean as well. It is therefore a good candidate for the study of nonlocal effects and inhomogeneous turbulence. As an example, a simple nonlocal theory is presented in Section 6.

Because of the difficulty in analytic and numerical treatment, oceanographic inertial effects are routinely ignored. Typically, this assumption is accompanied by an argument that the inertial terms are small in comparison to the other terms in a particular region of the ocean. However, it will be demonstrated here that some effects of inertia, even at modest Reynolds numbers, should be considered as part of the oceanic general circulation. Some of these results have profound implications for the setup and analysis of ocean models and the interpretation of observational data. Our model allows detailed analysis of the effects of inertia of a western-intensified system with analogues of many inertial behaviors of the ocean. A brief summary of the important sections of this paper follows.

Eddies play a critical role in the boundary layer structure. When a sufficient sink of vorticity exists, the meridional change in planetary vorticity of the boundary current is balanced by the eddy flux divergence. This turbulent western boundary layer is an important feature of the circulation in this model. This boundary layer is what allows a western-intensified mean state even when the inertial boundary layer width is wider than the frictional boundary layer width. The structure of the boundary layer in the main region of eddy activity is explored in Section 3.

In Section 4, it is noted that some of the solutions with quite different viscosities have very similar time-mean circulations. These homoparic calculations are quite common. The existence of these homoparic calculations may make it more difficult to correctly choose a viscosity distribution, but in practice they can be quite useful for the study of eddies under different viscosities.

The temporal behavior of the model contains both resonant and nonlinear interactions, and the latter makes the spectrum broad as the interior viscosity decreases. The temporal behavior of the less viscous calculations is discussed in Section 5. Particular attention is paid to the resonant modes, as this features prominently in the theory of the counterrotating gyres in Section 6. An empirical orthogonal function analysis gives insight into the nature of the spatial structure and temporal behavior of these modes. The details of the analysis methods are presented in the Appendix.

The deviations from Sverdrup (1947) balance in the basin interior, including interior closed circulations (counter-rotating gyres), are discussed in Section 6. Regions in which 
the time-mean circulation rotates in a sense counter to the wind forcing (as in Greatbatch and Nadiga, 1999; Özgökmen and Chassignet, 1998) are common in the less viscous calculations. Section 6 contains diagnosis of the balance of terms, directed numerical calculations, and an analytic calculation. This calculation (following Pedlosky, 1965b) demonstrates that the counter-rotating gyres that appear in the Eulerian mean are effectively explained by the nonlinear interaction in the interior of basin modes generated near the western boundary. This theory accounts for the seemingly required up-gradient mixing and the dependence of the counter-rotating gyres on the frequencies of the boundary current instabilities. The final section summarizes the results and discusses the relevance of these results to other models and ocean observations.

\section{Model}

As in Fox-Kemper and Pedlosky (2004), the model results presented here are from a $257 \times 257$ Chebyshev polynomial pseudo-spectral numerical barotropic model in a rectangular basin with spatially-variable viscosity to roughly parameterize boundary physics not directly represented in the model. The nondimensional equations governing the model are:

$$
\begin{gathered}
\frac{\partial \zeta}{\partial t}+\nabla \cdot\left(\hat{\mathbf{x}} \psi+\delta_{I}^{2} \mathbf{u} \zeta-\delta_{M}^{3} \nabla \zeta+\delta_{S} \nabla \psi\right)=-\sin (\pi y), \\
\zeta=\nabla^{2} \psi, \\
\delta_{M}^{3}=\frac{\delta_{I}^{3}}{\operatorname{Re}_{\mathrm{i}}}+\left(\frac{\delta_{I}^{3}}{\operatorname{Re}_{\mathrm{b}}}-\frac{\delta_{I}^{3}}{\operatorname{Re}_{\mathrm{i}}}\right)\left(e^{-x / \delta_{d}}+e^{-(1-x) / \delta_{d}}\right), \\
\delta_{d} \equiv \frac{\delta_{I}}{\sqrt{\operatorname{Re}_{\mathrm{i}}}} .
\end{gathered}
$$

The variables $\psi$ (streamfunction: $\mathbf{u} \equiv[-(\partial \psi / \partial y),(\partial \psi / \partial x)])$ and $\zeta$ (relative vorticity) are determined during integration. The initial conditions are resting $(\psi=0)$. The boundary conditions are slip $(\zeta=0)$ on the northern and southern 'fluid' boundaries and no-slip $[(\partial \psi / \partial x)=0]$ on the eastern and western 'solid' boundaries, as well as impermeability ( $\psi=0$ on the boundary). The basin is rectangular with $y$ between 0 and 1 and $x$ between 0 and $x_{e}$. The calculations are differentiated by the values of the constant parameters: $\delta_{I}$ (Charney, 1955, inertial boundary layer width), $\delta_{S}$ (Stommel, 1948, frictional boundary layer width), and $\mathrm{Re}_{\mathrm{i}}$ and $\mathrm{Re}_{\mathrm{b}}$ (boundary-layer Reynolds numbers for the interior viscosity and the boundary viscosity). For all of the calculations presented here, $\delta_{I}$ is 0.02 and $\delta_{S}$ is 0 , while $\mathrm{Re}_{\mathrm{i}}$ and $\mathrm{Re}_{\mathrm{b}}$ vary.

In this model it is sensible to define eddy as a perturbation from an Eulerian time mean. The time mean was taken over the last half of each integration (after equilibration), so that a Reynolds-averaged version of (1) may be analyzed: 


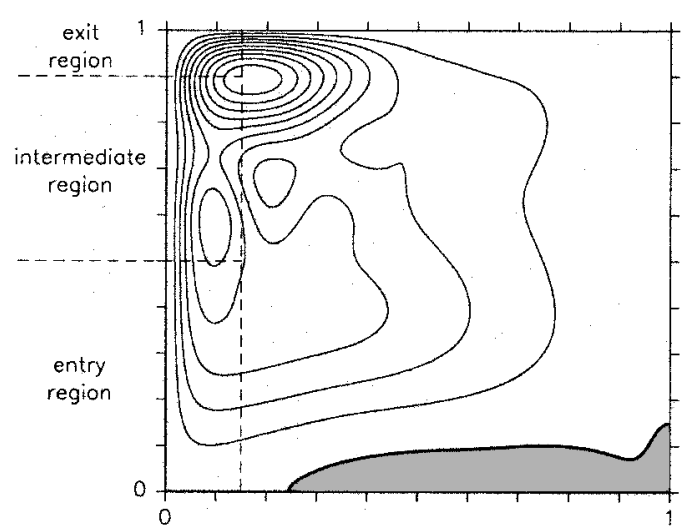

Figure 1. Contours of the time-mean streamfunction of the $\operatorname{Re}_{\mathrm{b}}=0.25, \mathrm{Re}_{\mathrm{i}}=5$ calculation with the averaging regions of the boundary layer indicated.

$$
0=\nabla \cdot\left(-\hat{\mathbf{x}} \bar{\psi}-\delta_{I}^{2} \overline{\mathbf{u}} \bar{\zeta}-\delta_{I}^{2} \overline{\mathbf{u}^{\prime} \zeta^{\prime}}+\delta_{M}^{3} \nabla \bar{\zeta}-\delta_{S} \nabla \bar{\psi}\right)-\sin (\pi y) .
$$

Overbar denotes time mean and primes denote perturbations from that mean. The primary effect of eddies in this model is given by the eddy flux convergence $\left(-\nabla \cdot \delta_{I}^{2} \overline{\mathbf{u}^{\prime} \zeta^{\prime}}\right)$. The terms will be called, respectively: $\beta$-flux convergence, mean flux convergence, eddy flux convergence, lateral friction flux convergence, bottom friction flux convergence, and wind forcing.

\section{Boundary layer balances}

Boundary-layer theories such as those of Stommel (1948), Munk (1950) and Charney (1955) propose a balance of terms in the vorticity equation near the western boundary. Figure 2 shows the meridional average (over different regions of the boundary layer) of the terms in (5) from one numerical calculation so that a comparison can be made with these theories. The averaging regions are indicated in Figure 1.

Figure 2a shows the meridional average in the region where the interior flow enters the boundary layer. In this region, the Charney (1955) inertial boundary layer solution can exist (as demonstrated by Greenspan (1962)) as relative vorticity from the basin interior is being imported into the boundary layer by the mean flow. On the seaward side of the boundary current (where the $\beta$-term still indicates a strong northward flow, $0.04<x<$ 0.08) the balance resembles the Charney (1955) model as the advection of planetary vorticity and relative vorticity are in balance, although in a region somewhat farther from the boundary than the Charney scale predicts. For $x<0.03$, there is a frictional sublayer acting to enforce the no-slip boundary condition. In the middle region, $0.02<x<0.08$, eddies widen the influence of the frictional sublayer by transporting vorticity from this region to the $0<x<0.02$ region. In essence, the solution in the entry region of the boundary layer agrees with the Il'in and Kamenkovich (1964) and Ierley and Ruehr (1986) 

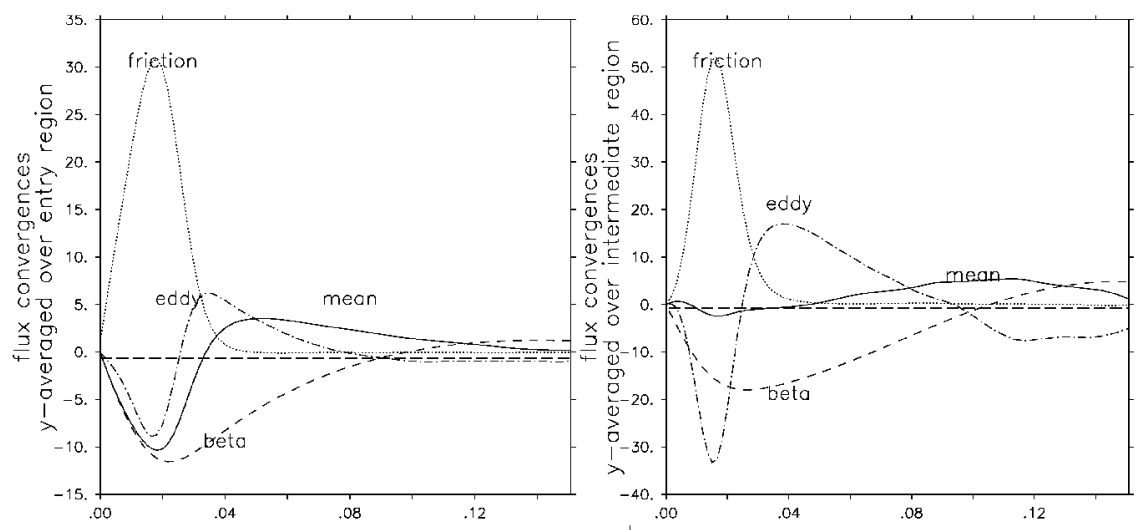

Figure 2. (a-b) show the meridional average of the vorticity flux convergences as a function of $x$ for the $\operatorname{Re}_{\mathrm{b}}=0.25, \mathrm{Re}_{\mathrm{i}}=5$ calculation. (a) shows the average over region (a) (the entry region). (b) shows the average over region (b) (the intermediate region).

steady-state picture of an inertial boundary layer with a frictional sublayer, except that the eddies join in to widen the influence of the frictional sublayer toward the interior.

Figure $2 b$ shows the meridional average of terms in (5) in the intermediate region. Here, the boundary layer structure is unlike any of the traditional steady-state theories. As in the western boundary current theory proposed by Pedlosky (1965a), the presence of time-dependent phenomena near the boundary is important, but here the critical feature is not the presence of reflecting remotely-forced Rossby waves; these eddies are locally generated by shear instabilities. The eddies play a critical role in energy and vorticity transport in this region, and they are present because the vorticity build-up near the boundary leads to shear instabilities.

On the interior side $(0.03<x<0.08)$ of the boundary current in Figure $2 \mathrm{~b}$, the balance is between eddy advection of vorticity and the advection of planetary vorticity, while closer to the boundary $(x<0.03)$ there is a frictional sublayer where friction balances both the eddy flux divergence and the $\beta$-flux divergence. It is interesting that just as the Charney (1955) inertial boundary layer theory fails, this alternative inviscid boundary layer structure forms.

Edwards and Pedlosky (1998) find a similar boundary layer structure in a model where cross-equatorial mass flux is required in a relatively inviscid boundary layer. In their model, a vorticity transformation is required for the flow to cross the equator. Edwards and Pedlosky (1998) find that at sufficiently large Reynolds number, the eddies transport vorticity from a relatively inviscid boundary layer to a frictional sublayer. The removal of the vorticity from the boundary layer fluid by the eddies changes the vorticity of the boundary layer fluid as it progresses.

While the boundary currents are similar here and in Edwards and Pedlosky (1998), the mass flux here occurs as a return flow for the Sverdrup (1947) flow, instead of being specified externally as in Edwards and Pedlosky (1998). Thus, this boundary layer results 
as part of the basin-wide vorticity budget rather than as a mass-flux condition. Unlike the Stommel (1948) and Munk (1950) layers, this boundary layer is not passive - the boundary-enhanced viscosity is required in this model for such a boundary-layer structure to exist rather than an inertially-dominated basin-filling recirculation (without a Sverdrup region) at Reynolds numbers much larger than one. Thus, in the presence of inertia, the boundary current must remove sufficient vorticity and flux sufficient mass for a Sverdrup interior to be satisfied. The exit region of the boundary layer is not shown as the two-dimensional recirculation gyre is not illuminated by a meridional average.

In summary, the boundary layer structure when $\mathrm{Re}_{\mathrm{i}}$ is greater than one and the solution is western intensified is: (1) similar to the Charney (1955) steady inertial boundary layer in the entry region, and (2) similar to the Edwards and Pedlosky (1998) eddy inertial boundary layer in the intermediate region, and (3) a recirculation gyre in the exit region.

\section{Homoparic calculations}

Throughout this paper and the companion paper (Fox-Kemper and Pedlosky, 2004), we emphasize the interchange of cross-streamline vorticity flux between frictional fluxes at low $\operatorname{Re}_{\mathrm{i}}$ and eddy fluxes at high $\mathrm{Re}_{\mathrm{i}}$. This is because we intended to demonstrate how the 'eddy viscosity,' that is the artificially high viscosity in the basin interior, could be lowered while maintaining a western-intensified mean flow (with the assistance of eddy vorticity fluxes). The companion paper demonstrates that this can be achieved by adjusting $\operatorname{Re}_{\mathrm{b}}$, thereby representing the effects of unresolved boundary processes. While calibrating this adjustment of $\mathrm{Re}_{\mathrm{b}}$, it became clear that some of the calculations with quite different values of $\mathrm{Re}_{\mathrm{i}}$ and $\mathrm{Re}_{\mathrm{b}}$ have very similar mean streamfunctions. For this reason, these calculations are called homoparic, for same average. The existence of homoparic equilibria adds a surprising twist to this picture; an $\mathrm{Re}_{\mathrm{b}}$-controlled circulation will not only be western intensified and have a similar time-mean kinetic energy to another calculation with larger $\mathrm{Re}_{\mathrm{b}}$ and smaller $\mathrm{Re}_{\mathrm{i}}$, but the entire shape and structure of the time-mean circulation will be similar. Two pair are examined in detail here. The first contains the $\operatorname{Re}_{\mathrm{b}}=0.5, \mathrm{Re}_{\mathrm{i}}=3$ calculation and the $\operatorname{Re}_{\mathrm{b}}=0.25, \mathrm{Re}_{\mathrm{i}}=5$ calculation. The second contains the $\operatorname{Re}_{\mathrm{b}}=3$, $\mathrm{Re}_{\mathrm{i}}=3$ calculation and the $\mathrm{Re}_{\mathrm{b}}=0.25, \mathrm{Re}_{\mathrm{i}}=8$ calculation. The time-mean streamfunctions of these calculations are shown in Figure 3.

Figure 4a illustrates that the contours of the maximum of the time-mean streamfunction connect not only the homoparic pairs shown in Figure 3, but entire families of homoparic equilibria sharing the same maximum streamfunction. All of the similar-looking mean streamfunctions in Figure 3 of the companion paper are connected by the contours of maximum streamfunction in Figure 4a.

While the time-mean circulations are quite similar in homoparic equilibria, the eddies must play a different role. Figure $4 \mathrm{~b}$ demonstrates that the homoparic pair members do not lie along contours of the Nusselt-like ratio of total vorticity flux to frictional flux (see Fox-Kemper and Pedlosky, 2004). The pair member with higher $\mathrm{Re}_{\mathrm{i}}$ has a larger Nusselt ratio, indicating that the eddies are doing more of the vorticity transport across the $\bar{\psi}=0.5$ 

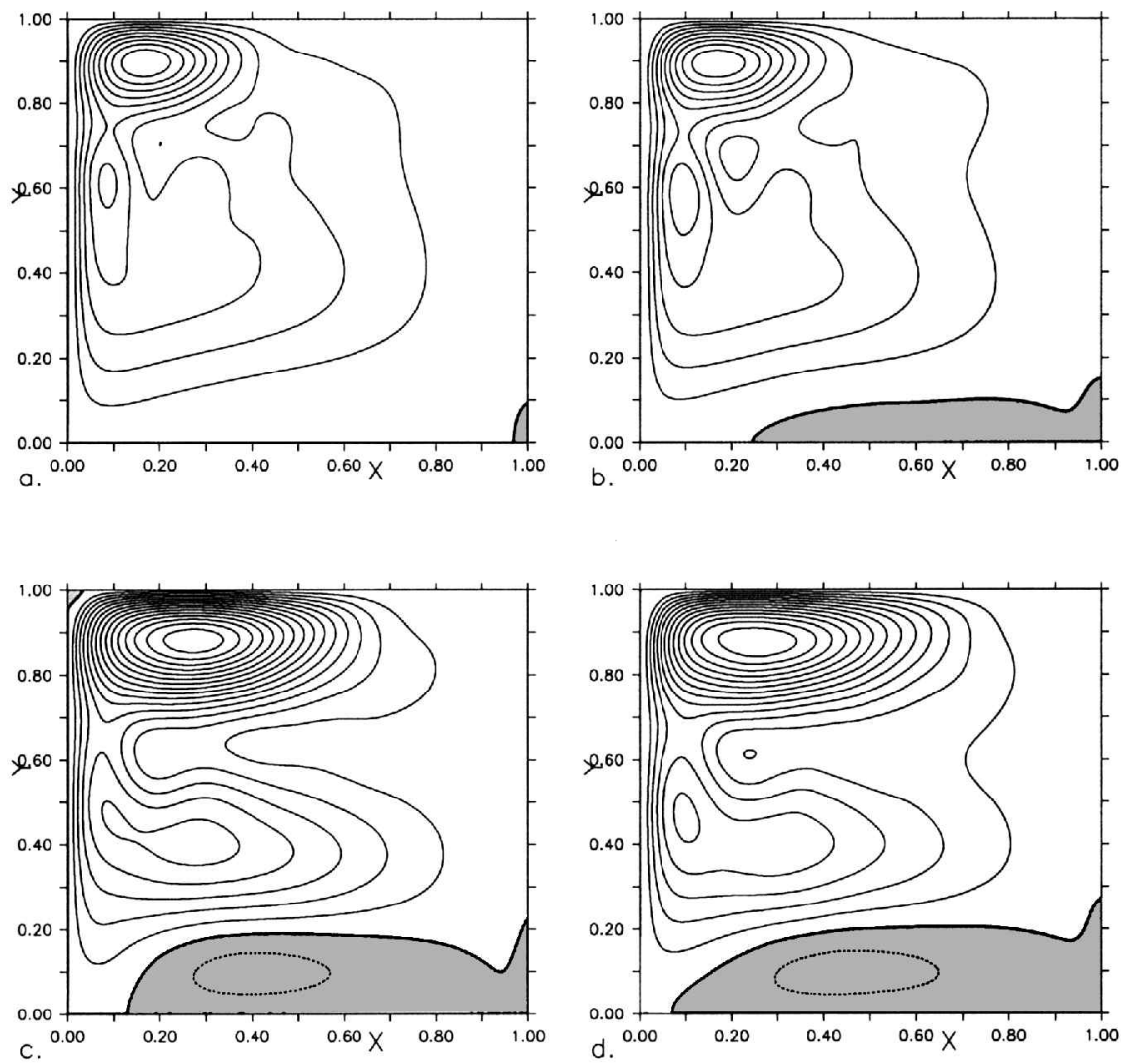

Figure 3. The time mean streamfunction contours of two pair of homoparic calculations. (a) shows the $\operatorname{Re}_{\mathrm{b}}=0.5, \mathrm{Re}_{\mathrm{i}}=3$ calculation and (b) shows the $\mathrm{Re}_{\mathrm{b}}=0.25, \mathrm{Re}_{\mathrm{i}}=5$ calculation. (c) shows the $\operatorname{Re}_{\mathrm{b}}=3, \mathrm{Re}_{\mathrm{i}}=3$ calculation and (d) shows the $\mathrm{Re}_{\mathrm{b}}=0.25, \mathrm{Re}_{\mathrm{i}}=8$ calculation. Contour interval 0.2, shaded regions are negative.

streamline. This streamline was chosen as a typical interior streamline, as it is halfway between the maximum and the boundary in the linear Munk solution. In fact, the eddies carry more of the vorticity flux across almost all of the streamlines (Fig. 5a). The members of a homoparic pair have the same input of vorticity by the wind and the same output of vorticity by friction (although with different vorticity gradients at the boundary). Thus, in order to have the same transport of vorticity across mean streamlines in the case with higher $\mathrm{Re}_{\mathrm{i}}$, the eddy flux must be correspondingly larger.

Figures $4 \mathrm{a}$ and $4 \mathrm{~b}$ indicate that lowering $\operatorname{Re}_{\mathrm{b}}$ will likely continue to control the circulation for much larger $\mathrm{Re}_{\mathrm{i}}$. The contours of maximum streamfunction curve sharply as $\mathrm{Re}_{\mathrm{b}}=0$ is approached, indicating that small changes in $\mathrm{Re}_{\mathrm{b}}$ will accommodate large changes in $\mathrm{Re}_{\mathrm{i}}$. Furthermore, the Nusselt ratio increases dramatically as $\operatorname{Re}_{\mathrm{b}}=0$ is approached for $\mathrm{Re}_{\mathrm{i}}$ larger than 3 ; the eddy fluxes become more important with decreasing $\mathrm{Re}_{\mathrm{b}}$ and increasing $\mathrm{Re}_{\mathrm{i}}$, just as required for continued control of the circulation. 


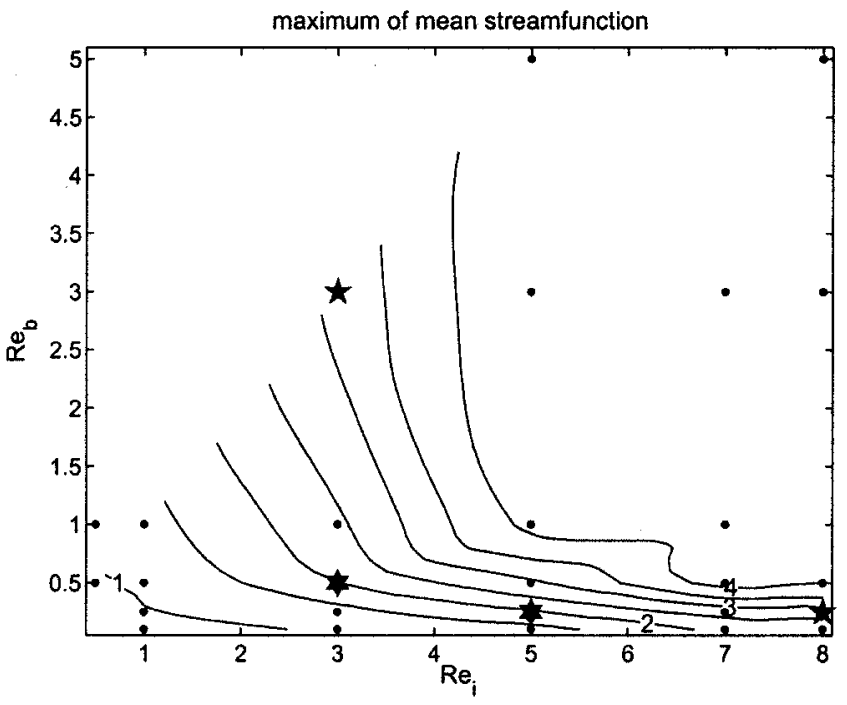

a.

total vorticity fluxdfrictional flux: $\mathrm{Nu}_{\zeta}(\psi=0.5)$

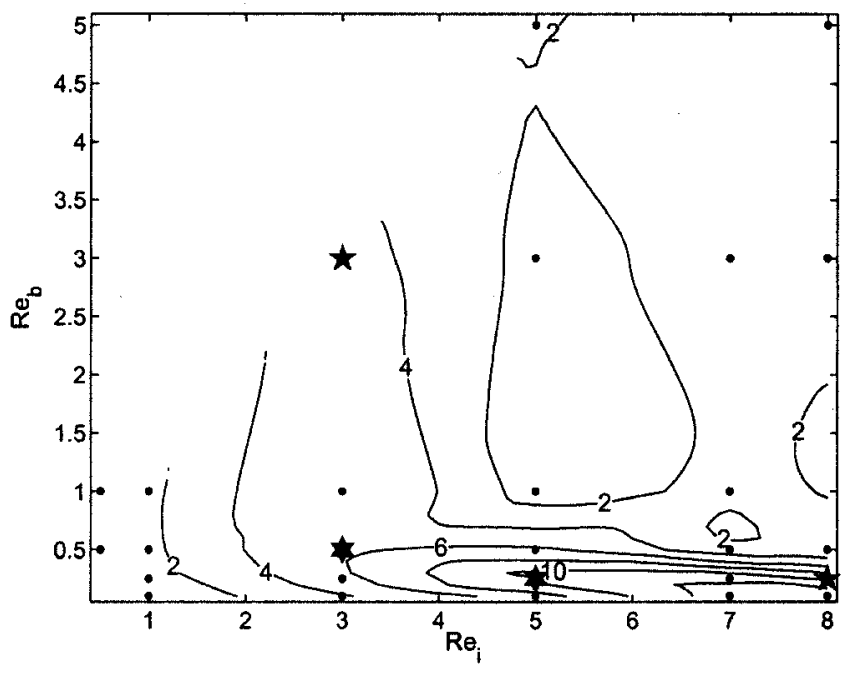

Figure 4. Contours of the (a) maximum value of the time-mean streamfunction and (b) $\mathrm{Nu}_{\zeta}(0.5)$, the ratio of the total vorticity flux to the frictional vorticity flux across $\bar{\psi}=0.5$ interpolated from the results of calculations with different $\mathrm{Re}_{\mathrm{b}}$ and $\mathrm{Re}_{\mathrm{i}}$. Dots indicate the location of the calculations used for the interpolation, and the homoparic pairs from the preceding figure are indicated with stars.

The eddy vorticity flux is quite different among the homoparic family members, because of small changes in the mean field, but mainly because of large changes in the eddies themselves. Figure 5b shows that there is significantly more variance in kinetic energy, 

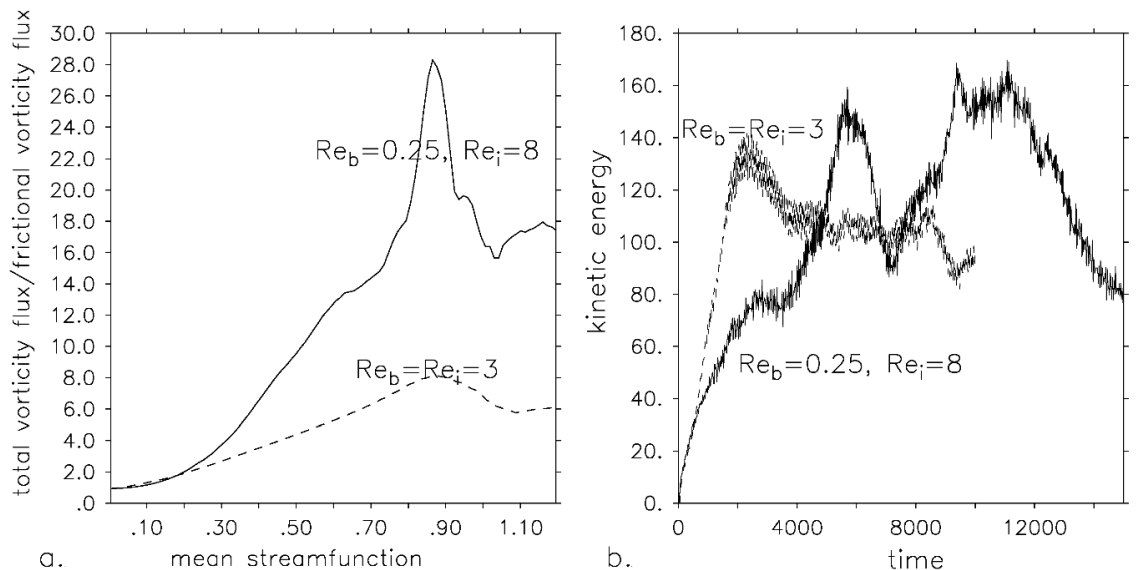

Figure 5. (a) The ratio $\mathrm{Nu}_{\zeta}$, the total vorticity flux to the frictional flux across a mean streamline, as a function of mean streamfunction for the $\operatorname{Re}_{\mathrm{b}}=\mathrm{Re}_{\mathrm{i}}=3$ and the $\mathrm{Re}_{\mathrm{b}}=0.25, \operatorname{Re}_{\mathrm{i}}=8$ calculations. (b) Total kinetic energy $\left(\iint|\nabla \psi|^{2} \mathrm{dA} / 2\right)$ versus time for the same calculations.

especially at low frequencies in the homoparic pair member with higher $\mathrm{Re}_{\mathrm{i}}$. Furthermore, the calculation with higher $\mathrm{Re}_{\mathrm{i}}$ takes significantly longer to spin up, presumably due to the balancing processes of the mean flow and the eddy fluxes formed by instabilities of the mean flow.

The location and magnitude of the eddy kinetic energy is also quite different between the two members in a homoparic pair (Fig. 6). Even though the time-mean streamfunction is similar, the perturbation variance is quite different.

A simple trade between the frictional flux and the eddy flux explains Figure 7a, so that the net effect of the eddy fluxes and frictional fluxes on the mean flow changes very little. However, the frictional flux must ultimately remove the vorticity input, so if there is a large
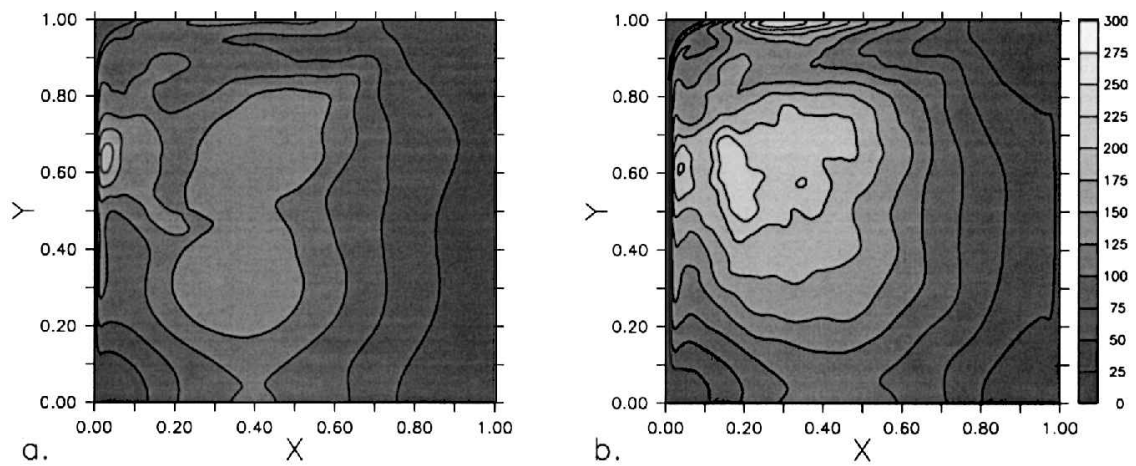

Figure 6. The time-mean eddy kinetic energy $\left(\overline{|\nabla \psi|^{2}} / 2\right)$ for the homoparic pair of (a) the $\operatorname{Re}_{\mathrm{b}}=\operatorname{Re}_{\mathrm{i}}=$ 3 calculation and (b) the $\operatorname{Re}_{\mathrm{b}}=0.25, \mathrm{Re}_{\mathrm{i}}=8$ calculation. 

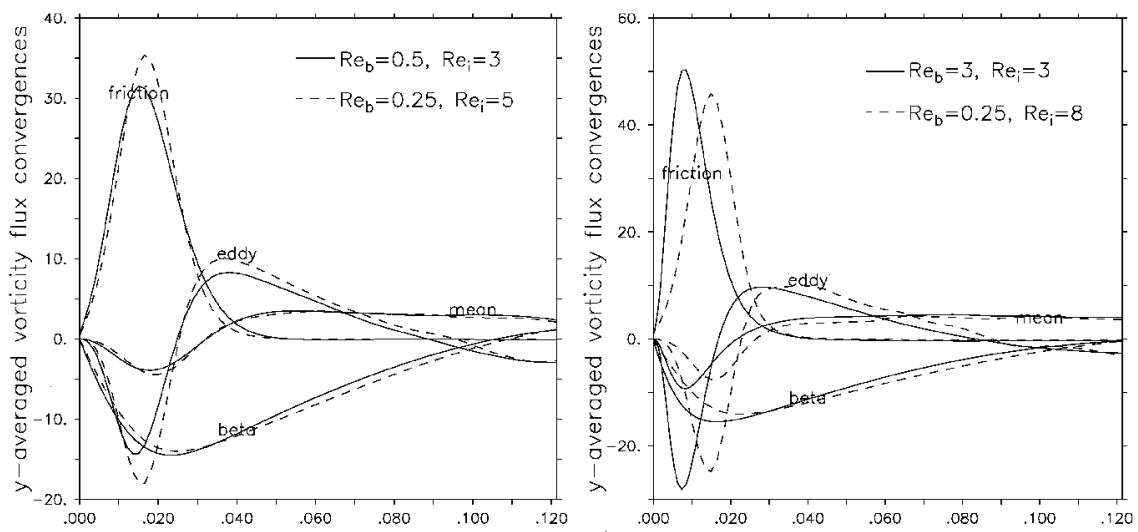

Figure 7. The meridional average of the terms in (5) for two pair of homoparic calculations.

change in $\mathrm{Re}_{\mathrm{b}}$ there must be a large change in the vorticity gradient at the boundary to compensate. Figure $7 b$ shows a homoparic pair where the changes in $\mathrm{Re}_{\mathrm{b}}$ and $\mathrm{Re}_{\mathrm{i}}$ are larger than those in the pair shown in Figure 7a. In Figure 7a it is the location of the mean fields which differs, rather than the magnitude. Along with the necessary change in the frictional flux convergence, the eddy, $\beta$, and mean flux divergences are located farther away from the boundary in the $\mathrm{Re}_{\mathrm{b}}=0.25, \mathrm{Re}_{\mathrm{i}}=8$ calculation.

In summary, homoparic equilibria exist over the whole parameter range studied. Their parametric dependence seems to imply a continuation of the control of the circulation proposed in the companion paper to much higher $\mathrm{Re}_{\mathrm{i}}$. It remains unclear why these equilibria have such similar time-mean flows, even though there are substantial differences between the eddies and boundary layer structure in the homoparic calculations. In the conclusion, some implications of the existence of homoparic equilibria for modeling and observational analysis are discussed.

\section{Temporal structure}

In a linear calculation model (e.g., Munk, 1950) a steady wind forcing always results in a steady solution. Even with variable wind forcing, the response of a linear system is easily predicted from a knowledge of the resonant modes of the system (per Pedlosky, 1965b). On the other hand, the moderately large $\mathrm{Re}_{\mathrm{i}}$ calculations presented here have a significant range of variability on many time scales, despite the fact that the forcing is steady. This section studies the nature of the instabilities, and attempts to connect it to a dynamical framework. Much of this variability is best characterized as resonant natural modes, and some of it appears forced by nonlinear interaction between modes. The interactions may produce frequencies that can be quite slow, or even steady.

Even within the small parameter range studied here, there is a diversity of temporal behaviors. As shown in Fox-Kemper and Pedlosky (2004), there is a qualitative difference 


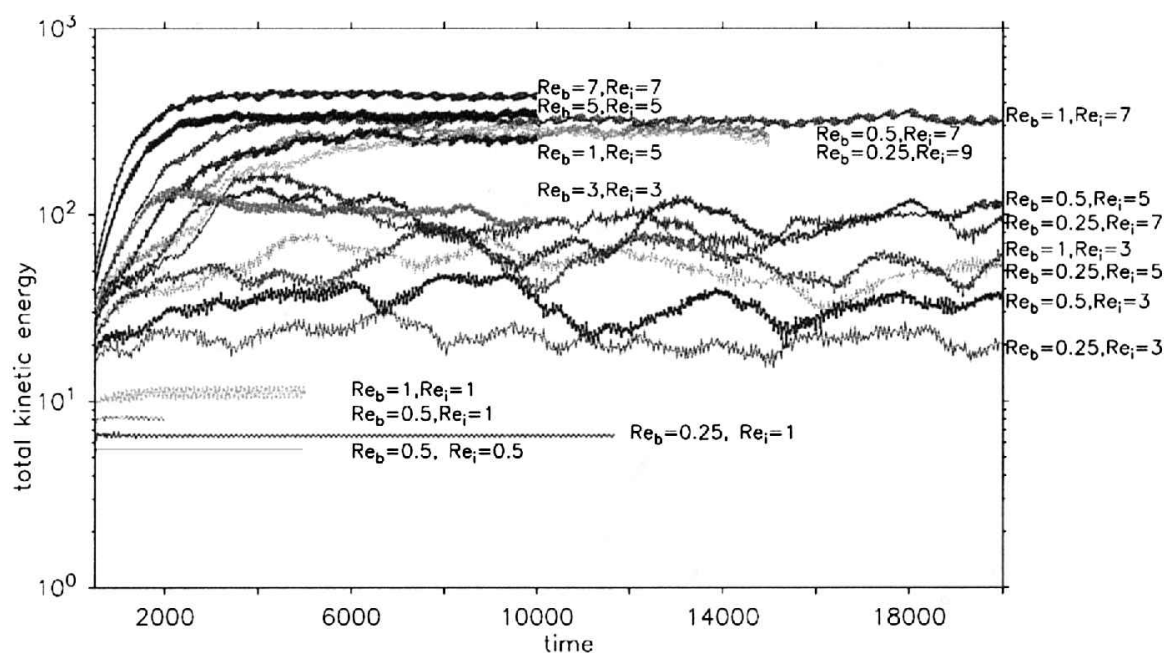

Figure 8. Total kinetic energy $\left(\iint|\nabla \psi|^{2} \mathrm{dA} / 2\right)$ versus time for different calculations.

between solutions that have a recirculation gyre which reaches the eastern boundary (inertially-dominated) and those that do not (western-intensified). There is also a qualitative difference in temporal behavior inertially-dominated and western-intensified calculations. The western-intensified solutions can be further divided into three categories: steady solutions, harmonic solutions, and chaotic solutions. Examples of the four cases are easily distinguished in Figure 8 (in order of increasing kinetic energy): steady, harmonic, chaotic, and inertially-dominated. The inertially-dominated calculations are higher in kinetic energy than the chaotic western-intensified calculations, but are proportionately less variable.

The (lower Reynolds number) steady and harmonic calculations are similar to the results found in other studies. The lowest Reynolds number calculation shown in Figure 8 is western intensified, relatively linear, and steady. At slightly higher Reynolds number (e.g., $\operatorname{Re}_{\mathrm{b}}=1, \operatorname{Re}_{\mathrm{i}}=1$ ), the mean flow remains western intensified even without boundaryenhanced viscosity, and the kinetic energy is seen to oscillate periodically with a few dominant frequencies. This is the regime where instabilities are present, but only of a few types, and they and their nonlinear interactions are strongly damped, so that secondary nonlinearly-forced interactions are weak. Sheremet et al. (1997) present an excellent examination of the eigenfunctions of linear perturbations about the steady-state solutions, and their stability and frequency in a very similar model. The finite amplitude variability in this range of Reynolds numbers agrees well with the eigenfunctions they present. In particular, the basin mode eigenfunctions (oscillatory modes related to the free, linear Rossby wave modes of the basin, here slightly damped and changed by the background flow) are active in these calculations at this Reynolds number and higher as we shall see below. 

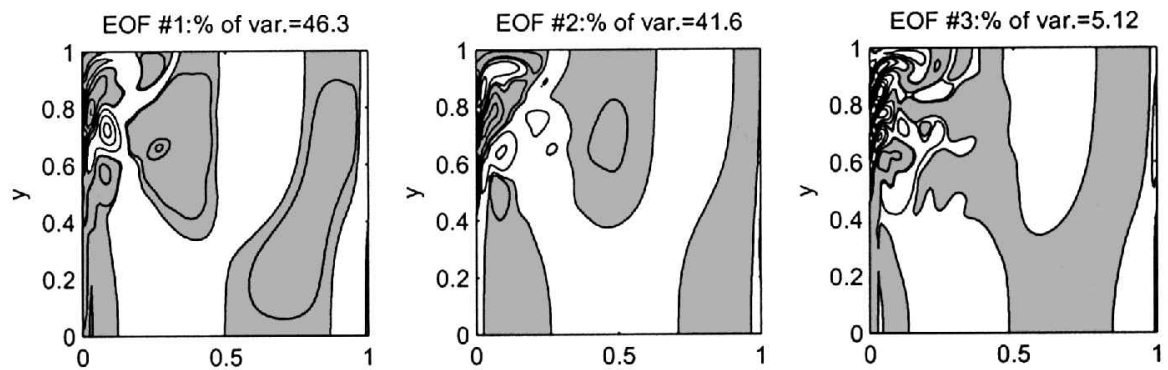

EOF \#4:\% of var. $=5.03$
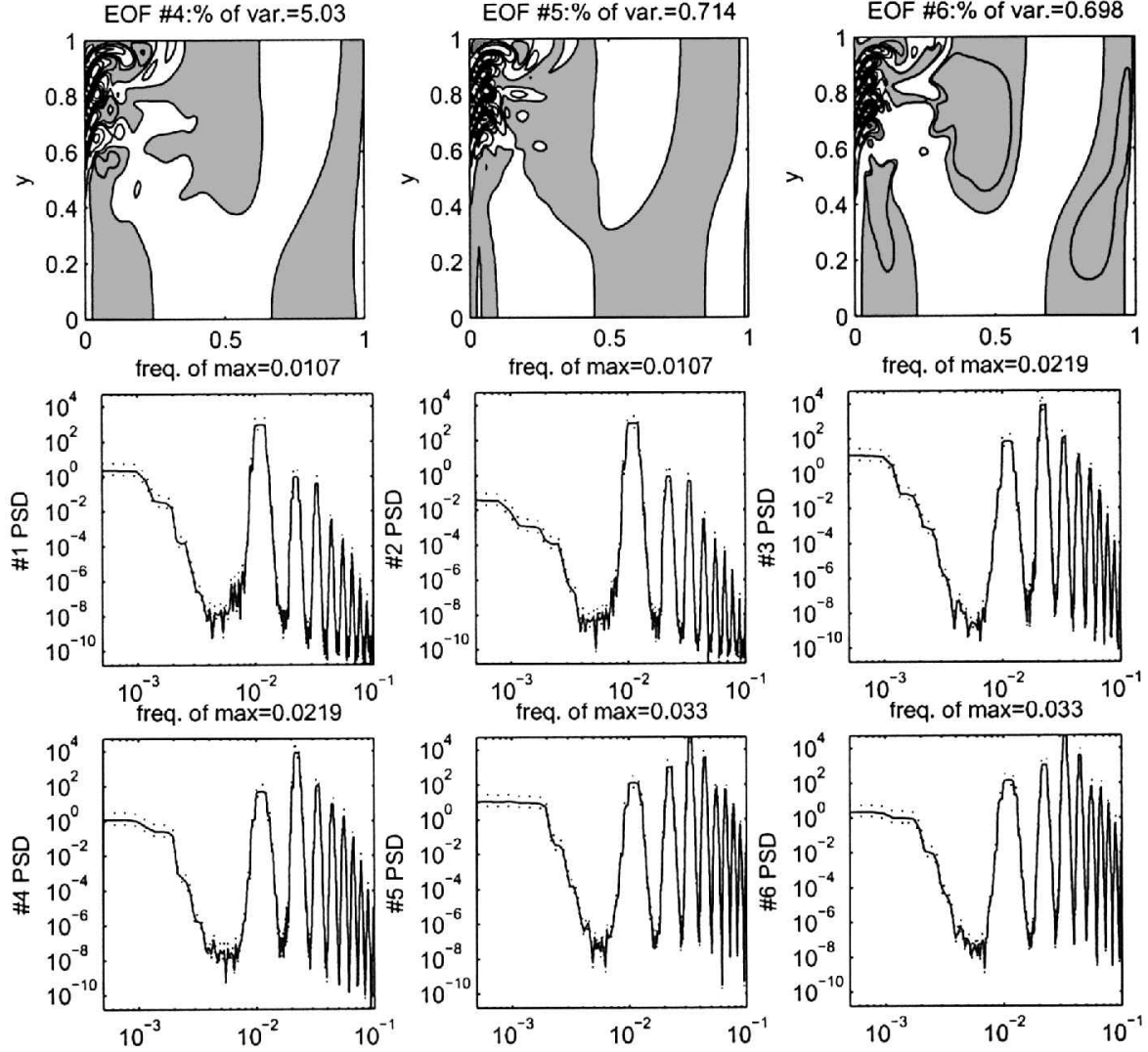

Figure 9. The upper two rows contour the EOFs of relative vorticity variability for the six largest variance $\mathrm{EOFs}$ for the $\mathrm{Re}_{\mathrm{b}}=1, \mathrm{Re}_{\mathrm{i}}=1$ calculation and give the percent of the total variance associated with each EOF. The lower two rows give an estimate of the PSD of the presence of each of the six EOFs, with an estimated $95 \%$ confidence interval (dotted).

To diagnose the primary modes of variability empirical orthogonal functions (EOFs) were calculated from the perturbation from the time mean of the streamfunction and vorticity. The details of the EOF analysis and some comments on the EOF representation of basin modes may be found in the Appendix. Figures 9 and 10 show the EOFs—and the 

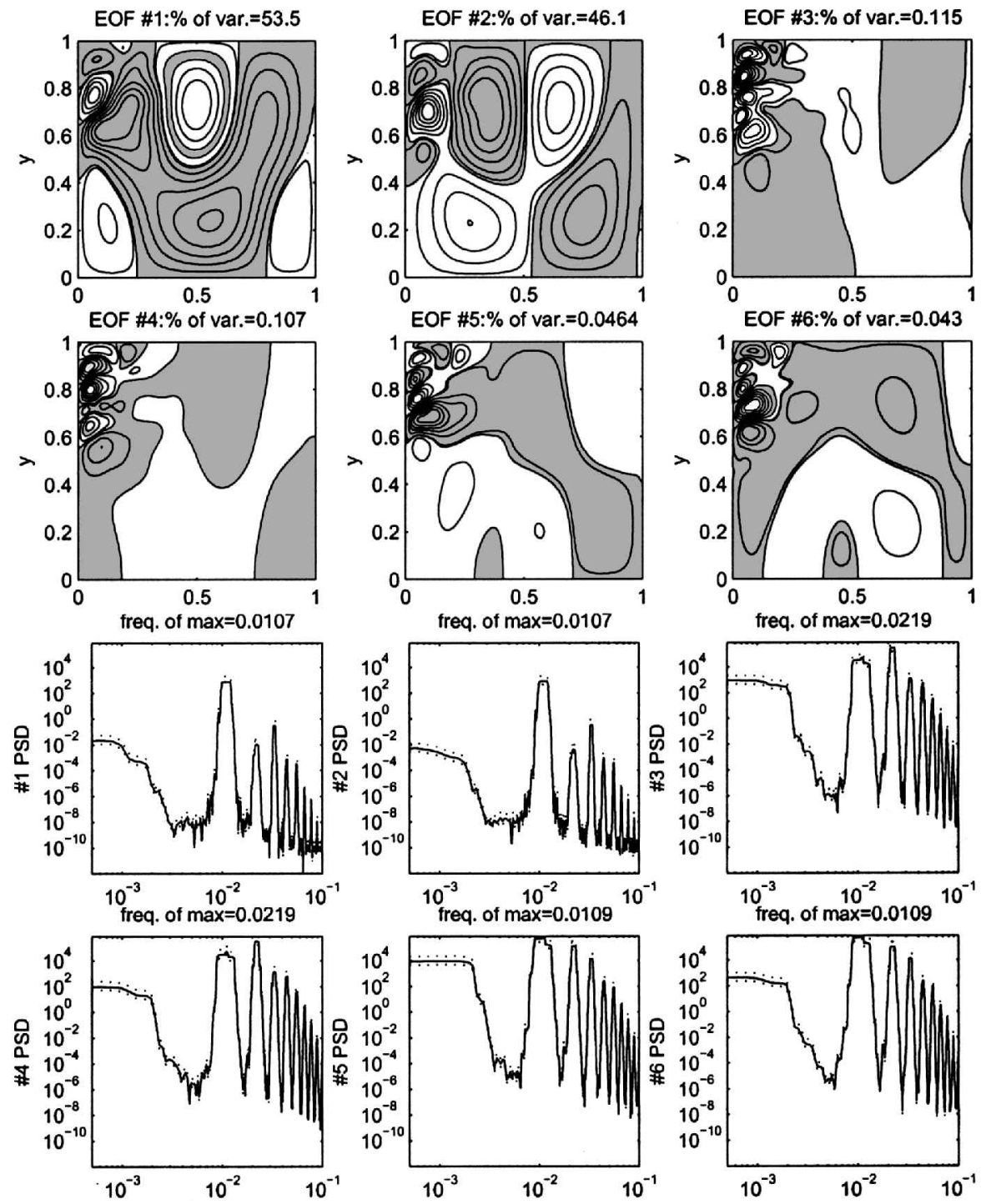

Figure 10. The upper two rows contour the EOFs of streamfunction variability for the six largest variance EOFs for the $\mathrm{Re}_{\mathrm{b}}=1, \mathrm{Re}_{\mathrm{i}}=1$ calculation and give the percent of the total variance associated with each EOF. The lower two rows give an estimate of the PSD of the presence of each of the six EOFs, with an estimated $95 \%$ confidence interval (dotted).

power spectrum density estimate (PSD) of the presence of each EOF-for the variability in $\zeta$ and $\psi$ about the time mean for the $\operatorname{Re}_{\mathrm{i}}=\mathrm{Re}_{\mathrm{b}}=1$ calculation.

Figure 9 reveals that most of the variance in the relative vorticity is contained very near the western boundary. Figure 10 shows that not all of the streamfunction variability is 
contained in the region near the boundary. In fact, most of the variance is in the first two EOFs, which are large-scale features. If, however, the projection of the streamfunction EOFs onto the first five linear basin modes is removed, the remaining pattern is concentrated in the boundary current or recirculation gyre region like the vorticity EOFs (not shown). Or, if one analyzes the streamfunction EOFs only within the boundary current region, structures of closed eddies like those in the vorticity EOFs emerge.

The vorticity is a function of higher derivatives of the streamfunction, and therefore the vorticity EOF is more strongly influenced by finer features, while the large-scale streamfunction variability dominates the streamfunction EOFs. Thus, the variability is divided into two types, small-scale features in the boundary current/recirculation gyre region, and large-scale features outside of this region. These features are easily detected with the vorticity and streamfunction EOFs respectively.

The large-scale features are quite wavelike. In fact, the two largest variance streamfunction EOFs closely resemble the expected spatial pattern of EOFs for the $(m, n)=(1,2)$ linear basin modes. Furthermore, their frequency is close to the linear, analytic basin mode frequency $(0.0107 \beta L$ versus $0.0113 \beta L)$. The first two vorticity EOFs are also at the same frequency, but do not resemble the relative vorticity pattern for a basin mode. Instead, the matching frequency suggests that the small-scale features in the boundary current region may be resonating the basin mode. If the basin mode is in a forced-dissipative balance, the relative vorticity pattern is indicative of the nature of the forcing. The results of Sheremet et al. (1997) suggest that there should be unstable eigenfunctions capable of resonating the basin modes, which tend to be more unstable than the basin modes themselves. Thus, the first two streamfunction EOFs and the first two vorticity EOFs indicate a basin mode resonated by near-boundary instabilities.

There are other modes present as well, even though the calculations are quite linear $\left(\operatorname{Re}_{\mathrm{i}}=\mathrm{Re}_{\mathrm{b}}=1\right)$. The remaining EOFs in Figure 10 are related to the wall-trapped eigenfunctions of Sheremet et al. (1997). The first four vorticity EOFs have the same frequencies as the first four streamfunction EOFs in Figure 10, and indicate that the small-scale features are all concentrated near the boundary.

The 5th and 6th vorticity EOFs with frequency $0.033 \beta L$ in Figure 9, however, are consistent with variability forced by the nonlinear interaction of the basin mode-like variability of the first and second EOFs and the wall-trapped instability of the 3rd and 4th EOFs. Consider the nonlinear interaction of the following two functions with simple dependence on $y$ and time, but complicated dependence on $x$.

$$
\begin{gathered}
\psi_{1}=f_{1}(x) \sin \left(\omega_{1}-l_{1} y\right), \\
\psi_{2}=f_{2}(x) \sin \left(\omega_{2}-l_{2} y\right), \\
J\left(\psi_{1}, \nabla^{2} \psi_{2}\right)+J\left(\psi_{2}, \nabla^{2} \psi_{1}\right)=g_{p}\left(x ; l_{1}, l_{2}\right) \sin \left[\left(\omega_{1}+\omega_{2}\right)-\left(l_{1}+l_{2}\right) y\right] \\
+g_{m}\left(x ; l_{1}, l_{2}\right) \sin \left[\left(\omega_{1}-\omega_{2}\right)-\left(l_{1}-l_{2}\right) y\right] .
\end{gathered}
$$


The functions $g_{p}$ and $g_{m}$ are complicated products of derivatives of $f_{1}$ and $f_{2}$ with coefficients depending on $l_{1}$ and $l_{2}$. However, the form of the nonlinear term depends on the original wavenumbers and frequencies quite simply — the resulting term either depends on the sums or the differences. This term will act as a forcing of two interaction modes, which will then depend on either the sums or the differences of wavenumber and frequency.

The sum of the frequency of the wall-trapped EOFs (\#3 and \#4, 0.0219 $\beta L$ ) and the frequency of the basin mode EOFs ( $\# 1$ and $\# 2,0.0107 \beta L$ ) is very close to the frequency of the 5th and 6th EOF in Figure $9(0.033 \beta L$ versus $0.0326 \beta L)$. Furthermore, the meridional scale of the interaction EOFs is smaller than the scale of either of the other EOFs, consistent with a sum of wavenumbers (under the rough approximation of first-order truncated Fourier transforms). Thus, it seems that these EOFs represent a nonlinear interaction of the other two modes.

The other large peaks in the PSD above the basin mode frequencies are also located at the sums and differences (and their sums and differences) of the two original frequencies to within the resolution of the PSD, likely indicating nonlinear interactions. This harmonic structure is characteristic of weakly nonlinear interactions. The Reynolds number is modest in these calculations, so the interaction modes are not as strong as the modes which generate them.

At higher Reynolds number (Fig. 11 and Fig. 12), the individual nonlinear interaction modes are no longer detectable. In these less viscous calculations, extended chains of nonlinear interactions occur and fill in the spectrum between the frequencies of linear instabilities and resonant modes. The PSDs of the EOFs show fewer peaks, and the signal to background ratio of those peaks is much smaller.

The fifth EOF of Figures 11 and 12 is very low frequency, and may indicate nonlinear forcing of the Sheremet et al. (1997) recirculation gyre mode. Based on their analysis, the presence of the zero-frequency recirculation gyre eigenmode indicates the existence of multiple steady solutions. The parameters of this calculation are close to the boundary for multiple steady solutions, and the appearance of this mode may indicate the existence of multiple equilibria in the time-dependent problem. The shape of the fifth EOF in Figure 12 indicates that the low frequency variability in this calculation (see also Fig. 8) can be attributed to changes in the structure and location of the recirculation gyre. Presumably, the pooling and draining of vorticity from the closed streamlines of the recirculation gyre in the balance between wind forcing and eddy fluxes is associated with this variability. The low-frequency variability may be a signal that the solution is traveling between the neighborhoods of multiple steady state solutions. An analysis similar to that performed by Meacham (2000) would be revealing as to the reason why the variance of the chaotic, western-intensified calculations with boundary enhanced viscosity is so much larger than the variance in the inertially-dominated calculations (see Fig. 8), but this is beyond the scope of this investigation.

However, some frequency peaks remain in many of the PSDs even at higher $\mathrm{Re}_{\mathrm{i}}$. The 

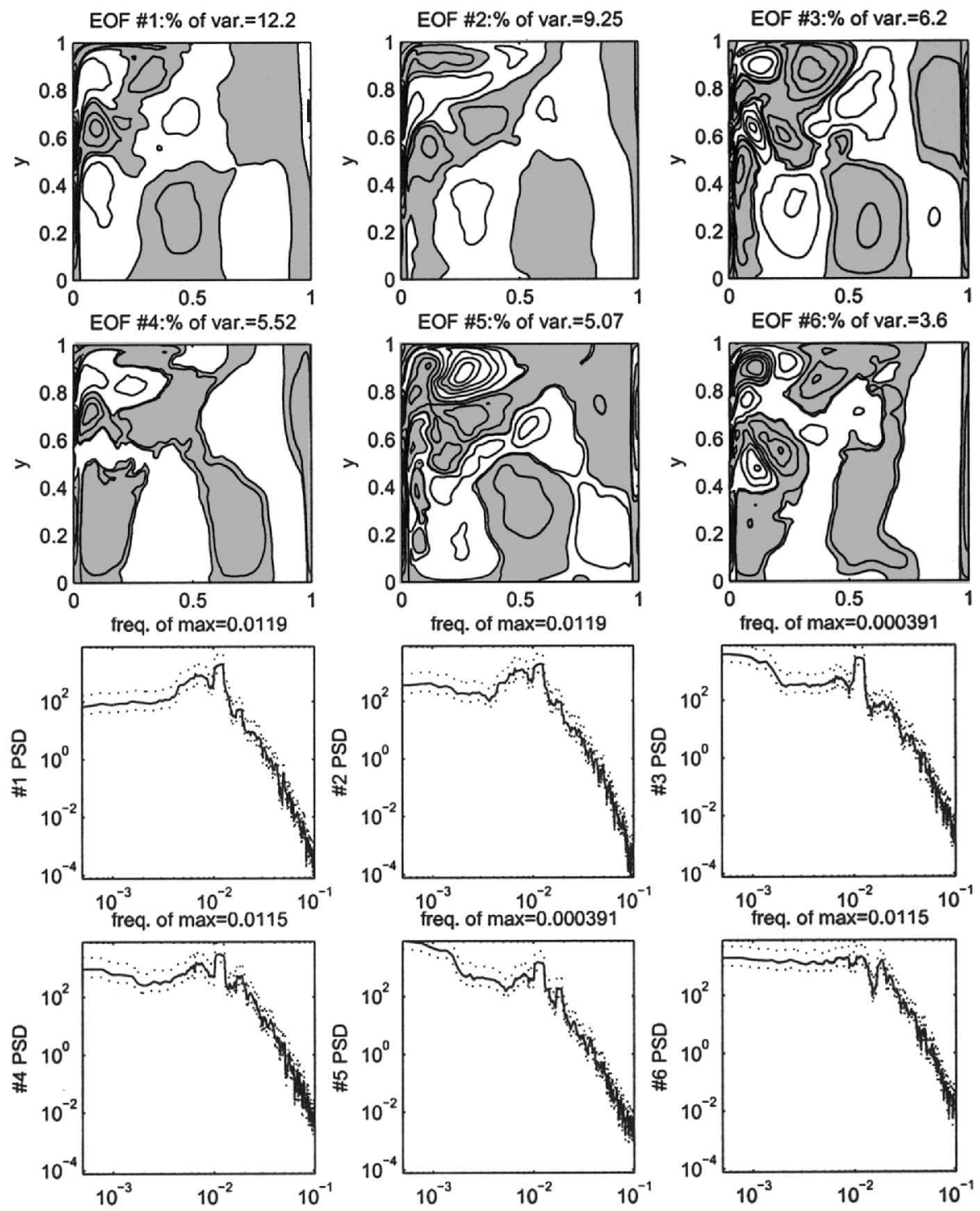

Figure 11. The EOFs of relative vorticity variability as in Figure 8 but for the $\operatorname{Re}_{b}=0.25, \operatorname{Re}_{\mathrm{i}}=5$ calculation which has much low-frequency variability.

streamfunction EOFs remain strongly peaked (Fig. 12). The strong peaks are related to the presence of basin modes. All of the EOFs shown except the fifth represent basin mode variability. Even in this strongly nonlinear calculation, more than $60 \%$ of the basin-average variance can be attributed to basin modes.

To test whether these EOFs truly represent basin modes, and to confirm the roles of 

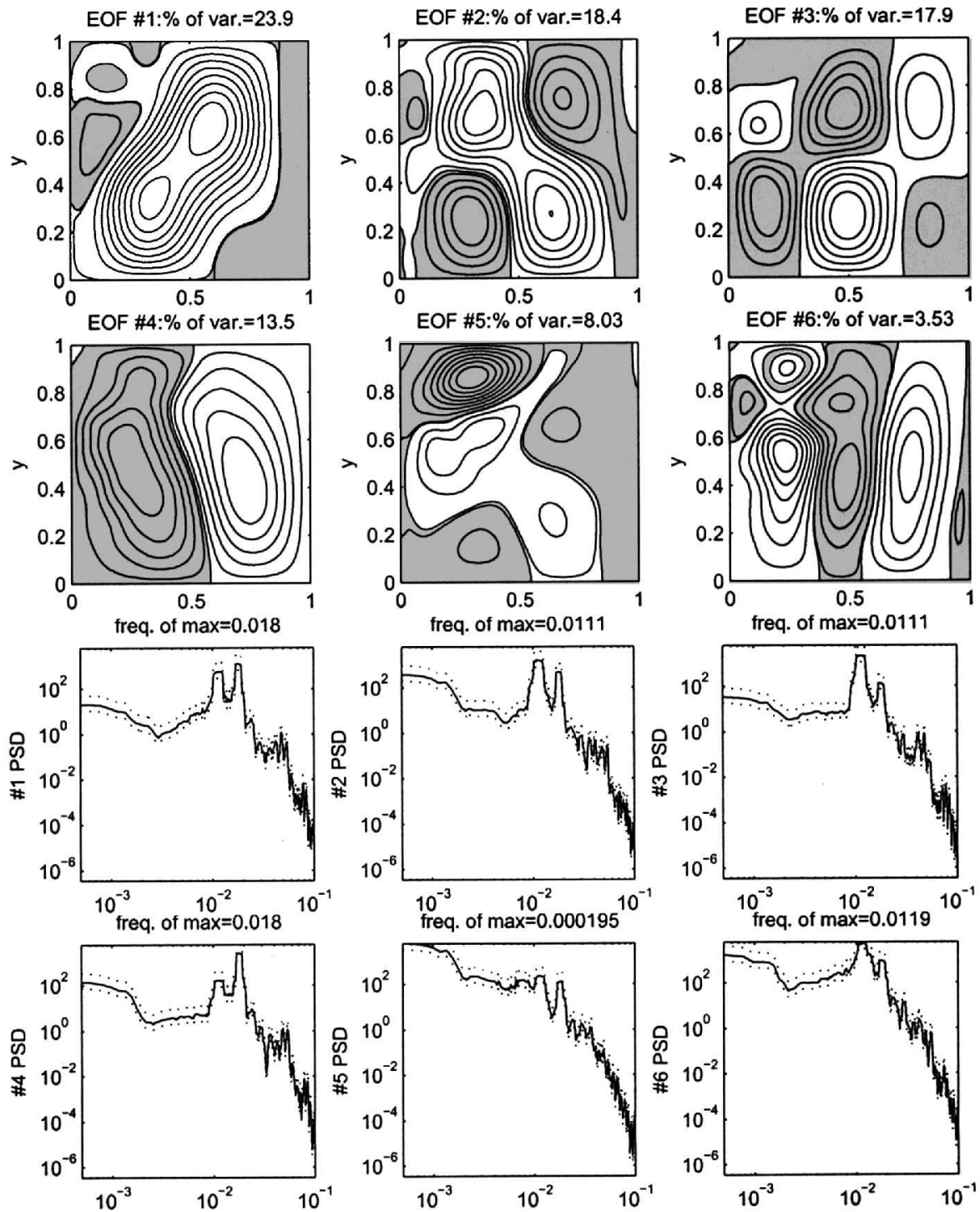

Figure 12. EOFs of streamfunction variability as in Figure 9 but for the $\operatorname{Re}_{\mathrm{b}}=0.25, \operatorname{Re}_{\mathrm{i}}=5$ calculation.

boundary current instability and resonating basin modes, a simple test was performed. Calculations in basins of different sizes were performed. By varying the basin width from $200 \%$ to $40 \%$ while arranging the wind forcing to produce a boundary current of the same transport and width as in Figures 9 and 10 (see Fox-Kemper (2003) for details) the reflection of waves at the eastern boundary was changed while leaving the western boundary current relatively unchanged. 
The relationship between the boundary current instabilities and the basin modes strongly affects whether modes are excited. Since the basin modes in the short basin are all lower frequency than the boundary instabilities, no basin modes result. ${ }^{3}$ In the short basin the highest basin mode frequency is $0.0094 \beta L$ for the gravest mode, and the lowest frequency instability in that calculation occurs near $0.0125 \beta L$.

The relationship between the boundary current instabilities and the basin modes strongly affects which modes are excited and how strongly they are excited. The first two EOFs of Figure 10 (the square basin) resemble the $(1,2)$ linear basin mode. In Figure 13 (the long basin), the first two streamfunction EOFs resemble the $(3,2)$ basin mode and the 3rd is similar to the $(1,2)$ basin mode. Weaker EOFs also resemble the basin modes in standing wave pattern and frequency. The frequencies of dominant boundary current vorticity EOFs are nearly constant with regard to the basin dimension $(0.0107 \beta L$ and $0.0219 \beta L$ for the square basin versus $0.0109 \beta L$ and $0.023 \beta L$ for the long basin). However, different basin modes are excited with different amplitudes because the resonant frequencies change with the basin dimension. In the square basin, the free, linear basin modes corresponding to those excited have a frequency of $0.0113 \beta L$, while in the long basin the free, linear frequencies are $0.0101 \beta L$ and $0.0123 \beta L$. Thus, as is typical for forced oscillators, the basin modes have the same frequency as the forcing, but the amplitude of each mode depends strongly on how close the forcing frequency is to the resonant frequency.

While the short and long basin cases presented here are at only $\operatorname{Re}_{\mathrm{i}}=\operatorname{Re}_{\mathrm{b}}=1$, a similar comparison with a long basin at $\mathrm{Re}_{\mathrm{b}}=\mathrm{Re}_{\mathrm{i}}=3$ (see Figs. 15 and 16) confirms that this result holds at higher Reynolds number as well. In fact, EOF analysis confirms that the basin modes continue to be the dominant EOFs of basin-wide streamfunction EOFs over the all Reynolds numbers explored here, but which basin modes are excited and by how much varies. Of course, the EOF analysis is biased somewhat in what it detects (see Appendix).

In summary, the single-gyre calculation is unsteady and temporally complicated, in contrast to the steady and linear solutions to the wind-driven problem. There are numerous modes of instability of the mean state, and these modes often resonate the basin modes and interact nonlinearly with each other. At low Reynolds number, EOF analysis does a good job of capturing almost all of the variance in modes resembling the eigenfunctions of linear perturbation studied by Sheremet et al. (1997). At higher Reynolds number, these interactions lead to a broadband spectrum for many of the EOFs and for the total kinetic energy. Over all of the parameter range studied here, however, most of the streamfunction variance was still in resonating basin modes.

\section{Basin interior balances and counter-rotating gyres}

Figure 3 reveals the presence of closed circulations in the southeast corner of the basin-a common result in the less viscous calculations shown here and in Fox-Kemper

3. There may be Rossby wave modes present as linear perturbations of the absolute vorticity gradient in the recirculation gyre, rather than the planetary vorticity gradient. 

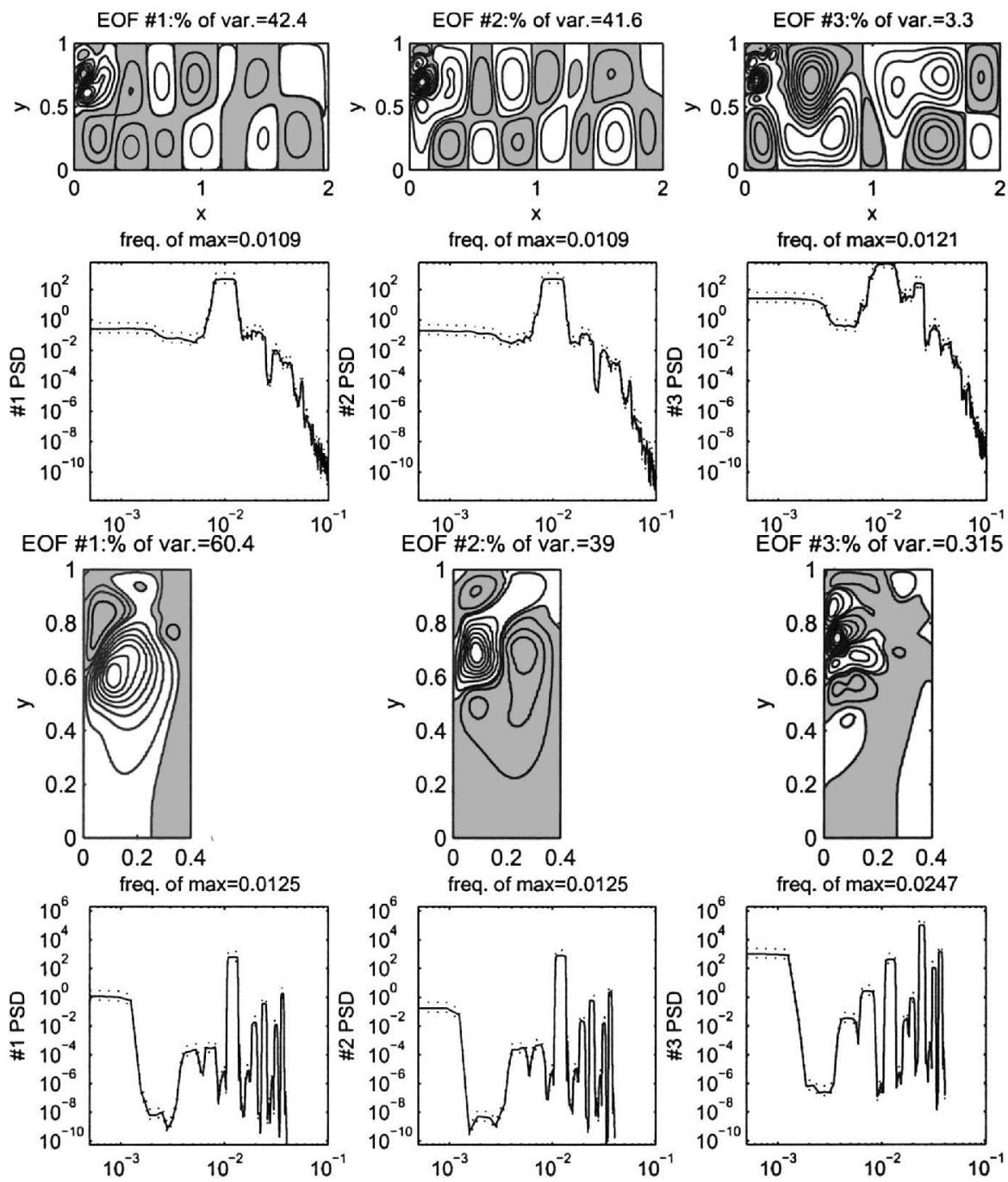

Figure 13. First 3 EOFs of streamfunction as in Figure 9 but for the $\operatorname{Re}_{b}=1, \operatorname{Re}_{\mathrm{i}}=1$ calculations in a long basin of zonal width 2 (upper 2 rows) and a short basin of zonal width 0.4 (lower 2 rows).

and Pedlosky (2004). The Eulerian-mean flow in these regions is in a direction opposite to that of the wind-stress; they counter-rotate. These counter-rotating gyres are present in the figures of Holland and Lin (1975); Kamenkovich et al. (1995); Sheremet et al. (1995), but are significantly weaker than those present here and go uncommented upon in the text of those papers.

Counter-rotating gyres are not present in linear calculations (where the Sverdrup relation is satisfied in this region) or in steady-state solutions of the traditional nonlinear problem 
with lateral friction and slip or no-slip boundary conditions (see, for example Ierley and Sheremet (1995)). In fact, Barcilon (1998) proves that such features cannot exist in the steady-state solutions of the inertial Stommel (1948) model. Almost all of the vorticity input within these mean streamlines is moved toward the boundary by eddies (see Fig. 9b of the companion paper where $\bar{\psi}$ is less than zero). During this process, the eddies and mean flow interact in such a way as to have an average circulation in a direction counter to the direction of the wind stress.

Greatbatch and Nadiga (1999) and Holm and Nadiga (2003) were perceptive in noting the importance of the appearance of counter-rotating gyres (which they call outer gyres) across double-gyre calculations with different boundary conditions and different friction operators. They propose parameterizations that cause similar gyres in coarse resolution calculations without eddies. In Greatbatch and Nadiga (1999), a parameterization of the effects of eddies is given in which the eddy parameterization fluxes vorticity down the mean gradient (equivalent to $\kappa \nabla\left(\delta_{I}^{2} \zeta+y\right)$ ) with a boundary condition that allows for no flux of absolute vorticity out of the basin. The solution which they obtain from this parameterization is steady, yet it possesses counter-rotating regions. This parameterization is not allowed in the models discussed here, as the single-gyre forcing has a net vorticity input which could not be balanced were the boundary condition no flux of absolute vorticity.

Salmon et al. (1976) propose that the most likely equilibrium statistical mechanical solution to the quasi-geostrophic equations is the one which maximizes entropy production. Griffa and Salmon (1989) were able to show that in a closed basin the maximal entropy production states for the unforced, non-dissipative calculation are modes resembling those of Fofonoff (1954). Counter-rotating regions found in other models have been attributed to such processes (e.g., Griffa and Castellari, 1991; Özgökmen and Chassignet, 1998). The counter-rotating gyres seen here do not appear to be of this variety, as the Fofonoff (1954) modes are primarily a balance between mean advection of vorticity and the $\beta$-term. The symmetry under $x \rightarrow-x$ of the equation for this balance leads to inertial boundary currents on both the eastern and western boundaries. The ansatz of the Fofonoff solution - that the absolute vorticity be a function of $\psi$-is also not satisfied in the counter-rotating region (see Fox-Kemper, 2003). The question of whether the counterrotating gyre represents the maximal entropy production solution remains open, but it is outside the scope of this investigation.

Figure 14 shows the meridional and zonal averages of the terms in (5) within the counter-rotating region. The counter-rotating gyres have a strong asymmetry from east to west and the primary balance of terms in (5) is wind forcing balanced by eddy flux convergence. The unforced, non-dissipative limit sought by Griffa and Salmon (1989) is thus not applicable here. This section therefore presents an alternative to the preceding theories, which is based on the prominence of resonating basin modes demonstrated in the last section and the primary balance of terms in the vorticity equation (e.g., Fig. 14).

Pedlosky (1965b) solves for the weakly-nonlinearinteraction of basin modes forced by a 

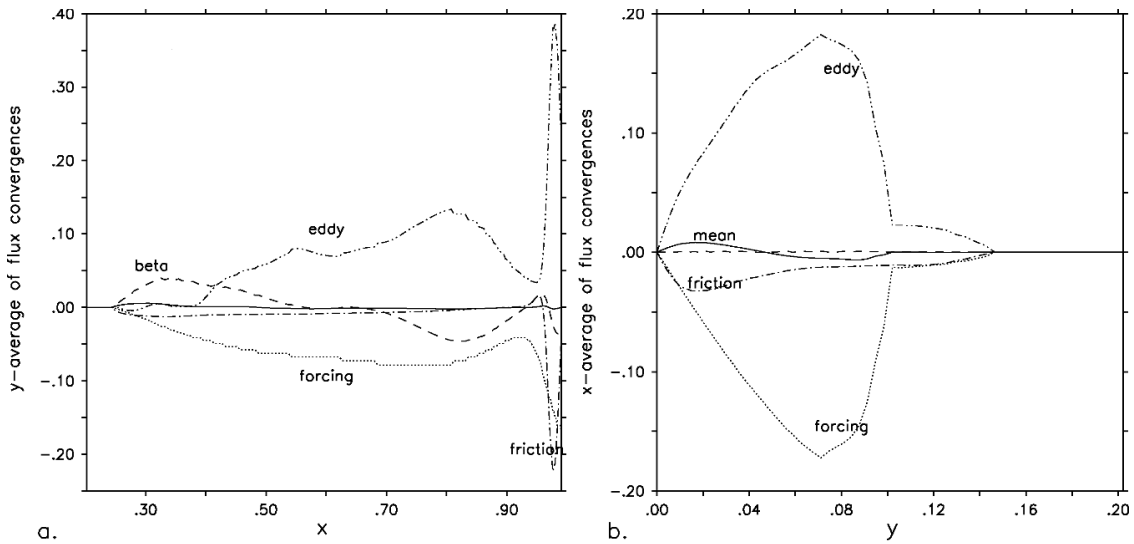

Figure 14. (a) and (b) show the meridional and zonal averages, respectively, of vorticity flux convergences in the region where $\bar{\psi}<0$ (the counter-rotating gyre) from the $\operatorname{Re}_{\mathrm{b}}=0.25, \operatorname{Re}_{\mathrm{i}}=5$ calculation.

traveling wave, $w_{E}=\cos (k x-\omega t) \sin (n \pi y)$, in a homogeneous model with bottom friction only. In the weakly nonlinear limit, the zeroth-order solution responds linearly to the wind forcing while the first-order solution contains a response to the nonlinear interaction of the zeroth-order solution. Pedlosky (1965b) demonstrates that the zerofrequency response to the nonlinear interaction can possess regions which rotate counterclockwise in the southern portion of the basin (for $n=1$ basin modes). However, Pedlosky (1965b) balances the eddy flux convergence with the $\beta$-term, whereas the counter-rotating regions here are in primary balance between the eddy flux convergence and the steady wind forcing with the $\beta$-term as a next-order correction (see Fig. 14). Regardless, the results of Pedlosky (1965b) show that a nonlinear interaction of basin modes can cause an Eulerian-mean circulation tantalizingly similar to the counter-rotating gyres. ${ }^{4}$

The eddy flux convergences and divergences in the region of the counter-rotating gyre (Fox-Kemper and Pedlosky 2004, Fig. 7b) have large spatial scale indicating basin-scale motions. Also, movies of the vorticity evolution reveal wave-like motion of absolute vorticity present in this region propagating from east to west, rather than coherent vortices. In the preceding section, it was demonstrated that much of the variance can be attributed to basin modes, and this is especially true in the eastern part of the basin where the counter-rotating gyres are.

A calculation was performed with a zonally elongated basin with wind forcing only in

4. The Lagrangian mean result will be quite different, as free waves do not affect the Lagrangian mean (Moore, 1970). What the Lagrangian mean in this forced-dissipative system will be is an interesting question, but outside the scope of this investigation. Briefly, note that since every fluid parcel is continually exposed to wind vorticity input, all parcels must eventually enter a frictional region to remove this vorticity. Since the Eulerian mean flow does not directly enter the frictional region, there must be a chaotic advection of Lagrangian fluid parcels into the frictional region. 

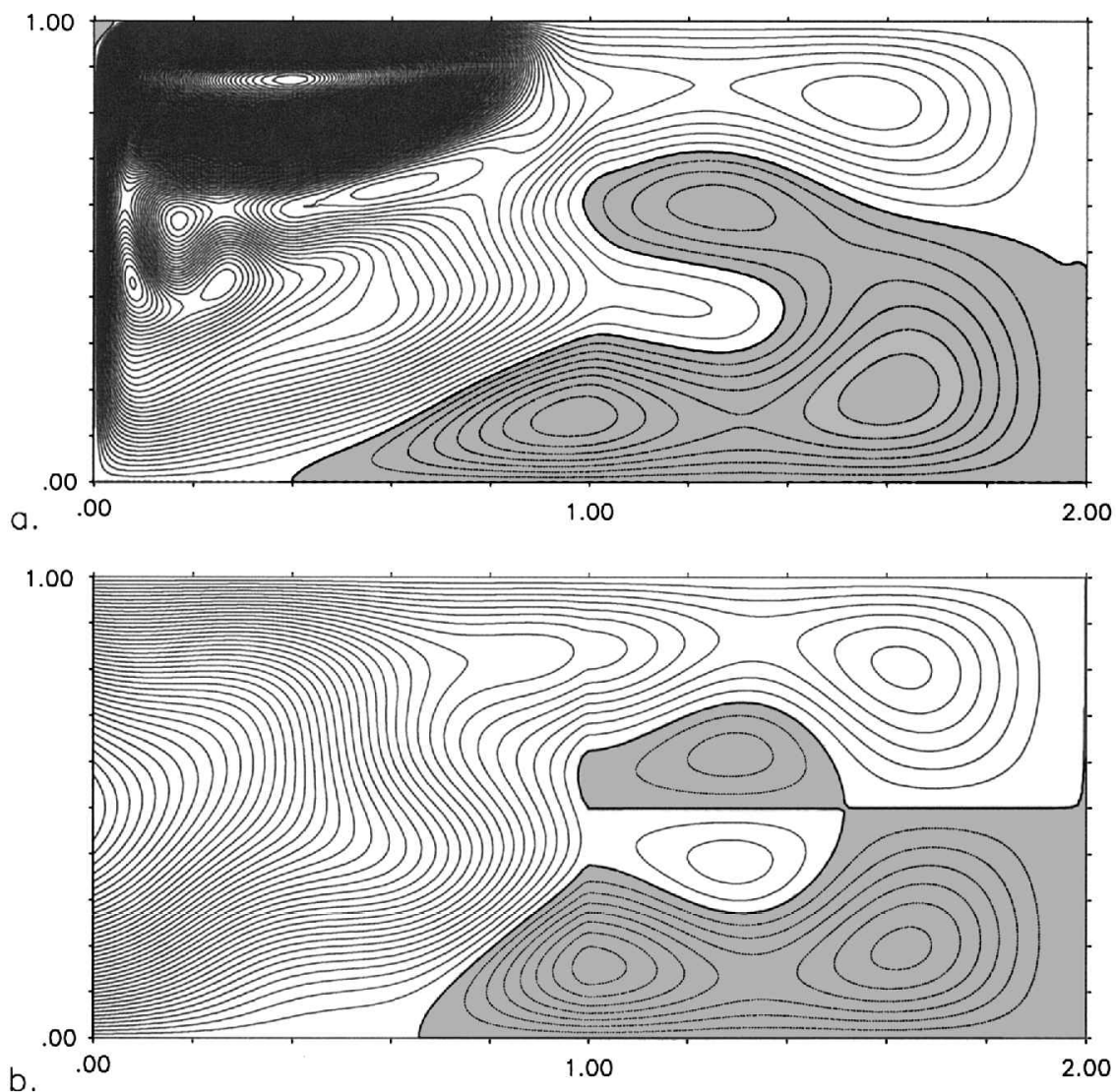

Figure 15. (a) The time-mean streamfunction showing the counter-rotatinggyres in a calculationin a elongated basin with $\operatorname{Re}_{\mathrm{b}}=3, \mathrm{Re}_{\mathrm{i}}=3$. There is wind forcing only in the western half of the basin. (b) The prediction for the interior time-mean streamfunction with analytic basin modes $(3,1)$, $(2,1),(1,2),(1,1)$, and $(2,2)$ with variances from EOF diagnosis. The contour interval is 0.025 , and regions of negative streamfunction are shaded.

the western half of the basin to demonstrate the importance of the basin modes in generating the counter-rotating gyres. In this calculation, the linear Munk solution extends only halfway across the basin, yet there are clearly Eulerian-mean circulations which occur in the eastern half of the basin where there is no wind forcing (Fig. 15a). In fact, there are regions which rotate counterclockwise as counter-rotating gyres do, and there are regions which rotate clockwise as well: co-rotating gyres.

Figure 16 shows the meridional and zonal averages of the terms in (5) in the counter-rotating region. In Figure 16a, only the westernmost part of the counter-rotating region experiences the wind forcing and the easternmost region is frictional. The middle of the counter-rotating region has a balance between the $\beta$-term and the eddy flux divergence, just as the resonant solutions in Pedlosky (1965b) do. 

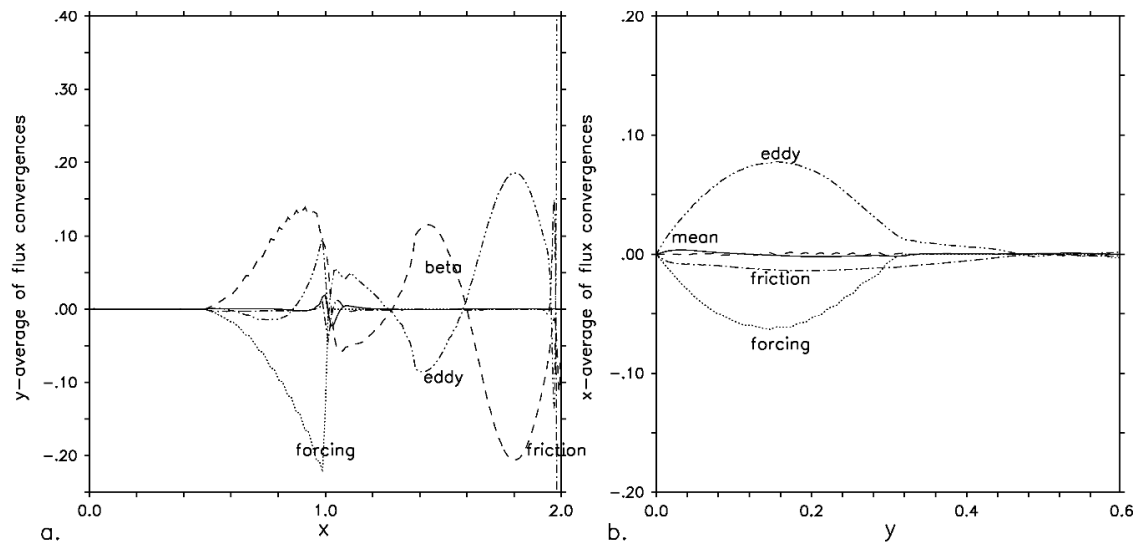

Figure 16. (a) and (b) show the meridional and zonal averages, respectively, of terms in (5) in the region where $\bar{\psi}<0$ (the counter-rotating gyre) from the $\operatorname{Re}_{\mathrm{i}}=\operatorname{Re}_{\mathrm{b}}=3$ elongated basin calculation with wind only in the western half of the basin.

A simple analytic model of the counter-rotating gyres synthesizes and clarifies some of these notions. Suppose that the sources of variability of the western boundary current and recirculation gyre are modeled simply by a periodic forcing in a region near the western boundary. As a useful simplification, bottom friction will be used instead of lateral friction. Note, however, that the western boundary layer with bottom friction will not produce instabilities; the instabilities of the boundary current are imposed by hand. The barotropic vorticity equation is

$$
\frac{\partial \nabla^{2} \psi}{\partial t}+\frac{\partial \psi}{\partial x}+\delta_{I}^{2} J\left(\psi, \nabla^{2} \psi\right)+\delta_{S} \nabla^{2} \psi=-\sin (\pi y)+A_{f} \sin \left(n_{f} \pi y\right) \cos \left(\omega_{f} t\right) e^{-x / \delta_{f}}
$$

The Jacobian is denoted $J(a, b) \equiv(\partial a / \partial x)(\partial b / \partial y)-(\partial b / \partial x)(\partial a / \partial y)$. The form of the forcing is chosen for simplicity and to resemble a term in a Fourier-Laplace-Fourier expansion of a more complex instability. For simplicity, only the square basin case will be calculated explicitly here.

Consider the weakly nonlinear perturbation series: $\psi=\psi_{0}+\delta_{I}^{2} \psi_{1}+\cdots$ under the assumption that $\delta_{I} \ll 1$. The zeroth-order equations will be linear, so the time-dependent and time-mean equations can be decoupled and solved independently. Once this is accomplished, the nonlinear interaction of the time-dependent, zeroth-order, linear solution will have a contribution to the first-order mean equation. It will be shown that including this correction to the zeroth-order (Sverdrup, 1947) mean flow gives excellent agreement to the counter-rotating gyres.

To begin, $\delta_{S}$ can be any magnitude. Thus, neglecting the inertial term,

$$
\frac{\partial \nabla^{2} \psi_{0}}{\partial t}+\frac{\partial \psi_{0}}{\partial x}+\delta_{S} \nabla^{2} \psi_{0}=-\sin (\pi y)+A_{f} \sin \left(n_{f} \pi y\right) \cos \left(\omega_{f} t\right) e^{-x / \delta_{f}} .
$$


This equation is linear, so it is uncoupled into a steady equation (similar to Stommel, 1948) and a time-dependent equation (similar to Pedlosky, 1965b).

$$
\begin{gathered}
\frac{\partial \overline{\psi_{0}}}{\partial x}+\delta_{S} \nabla^{2} \overline{\psi_{0}}=-\sin (\pi y), \\
\frac{\partial \nabla^{2} \psi_{0}^{\prime}}{\partial t}+\frac{\partial \psi_{0}^{\prime}}{\partial x}+\delta_{S} \nabla^{2} \psi_{0}^{\prime}=A_{f} \sin \left(n_{f} \pi y\right) \cos \left(\omega_{f} t\right) e^{-x / \delta_{f}} .
\end{gathered}
$$

First guesses at the solutions gives the interior solution (Sverdrup, 1947) and a particular solution to the time-dependent equation.

$$
\begin{gathered}
\overline{\psi_{0}} \approx(1-x) \sin (\pi y), \\
\psi_{0}^{\prime} \approx \mathscr{R}\left[\varphi_{p} e^{\left(-x / \delta_{f}\right)} e^{i \omega_{f} t} \sin \left(n_{f} \pi y\right)\right], \\
\varphi_{p} \equiv \frac{A_{f}}{i \Omega_{f}\left(\delta_{f}^{-2}-n^{2} \pi^{2}\right)-\delta_{f}^{-1}}, \\
\Omega_{f} \equiv \omega_{f}-i \delta_{s} .
\end{gathered}
$$

The real part is denoted by $\mathscr{R}$.

The boundary conditions are imposed by addition of homogeneous solutions. This results in somewhat complicated formulas. The steady solution is the Stommel (1948) solution for the steady problem, valid for all values of $\delta_{S}$, (17). The time-dependent solution for all values of $\delta_{S}$ is (18).

$$
\begin{gathered}
\overline{\psi_{0}}=\frac{\sin (\pi y)}{\pi^{2} \delta_{S}}\left[\frac{1-e^{B}}{e^{A}-e^{B}} e^{A x}-\frac{1-e^{A}}{e^{A}-e^{B}} e^{B x}-1\right], \\
A \equiv \frac{-1+\sqrt{1+4 \pi^{2} \delta_{S}^{2}}}{2 \delta_{S}}, B \equiv \frac{-1-\sqrt{1+4 \pi^{2} \delta_{S}^{2}}}{2 \delta_{S}} . \\
\psi_{0}^{\prime}=\mathscr{R}\left[\varphi_{p} \sin \left(n_{f} \pi y\right) e^{i \omega_{f} t}\left(e^{\left(-x / \delta_{f}\right)}+\frac{\sin (\alpha(x-1))}{\sin (\alpha)} e^{i x / 2 \Omega_{f}}-\frac{\sin (\alpha x)}{\sin (\alpha)} e^{-\left(1 / \delta_{f}\right)+\left[i(x-1) / 2 \Omega_{f}\right]}\right)\right], \\
\alpha \equiv \frac{\sqrt{1-4 n_{f}^{2} \pi^{2} \Omega_{f}^{2}}}{2 \Omega_{f}} .
\end{gathered}
$$

At the next order, nonlinearity couples the different frequencies (and introduces the possibility of a continuous frequency spectrum of singular non-normal modes, which will hereafter be ignored for simplicity). The counter-rotating gyres are a feature of the time-mean flow, so we consider only the Reynolds-averaged equation for the first-order. Secular modes do not appear to be a problem as the nonlinear interaction terms are not proportional to other basin mode solutions. 


$$
\frac{\partial \overline{\psi_{1}}}{\partial x}+\delta_{S} \nabla^{2} \overline{\psi_{1}}=-\delta_{I}^{2} J\left(\overline{\psi_{0}}, \nabla^{2} \overline{\psi_{0}}\right)-\delta_{I}^{2} \overline{J\left(\psi_{0}^{\prime}, \nabla^{2} \psi_{0}^{\prime}\right)}
$$

Thus, the mean flow correction involves the mean advection of mean relative vorticity and the eddy advection of eddy relative vorticity. Using the solutions to the uncoupled linear problems, the leading-order nonlinear interaction can be determined.

By differentiating the steady solution valid for all $\delta_{S}$, (17), then taking limit of small $\delta_{S}$, the correct nonlinear product is formed. ${ }^{5}$ All of the nonlinear terms for the mean-mean interaction vanish exponentially outside of the boundary layer except for

$$
\delta_{I}^{2} J\left(\overline{\psi_{0}}, \nabla^{2} \overline{\psi_{0}}\right) \approx \frac{\delta_{I}^{2} \sin (2 \pi y)}{2 \pi^{3} \delta_{S}^{2}} A^{3} e^{-A+A x} \approx \frac{1}{2} \pi^{3} \delta_{S} \delta_{I}^{2} \sin (2 \pi y) .
$$

If the periodic forcing is resonant with a basin mode-that is, $\omega_{f}=\left(2 \pi \sqrt{m^{2}+n^{2}}\right)^{-1}$ for some integer $m$, and $n=n_{f}$ (as the basin modes are orthogonal in $y$ )—then to lowest order in $\delta_{S}$ the time-dependent solution becomes

$$
\psi_{0}^{\prime}=\mathscr{R}\left[\frac{4 \pi i m \varphi_{0} \omega_{f}^{3}}{\delta_{S}} \sin (m \pi x) \sin (n \pi y) e^{i \omega_{f} t+\left(i x / 2 \omega_{f}\right)}\left(-1+(-1)^{m} e^{-\left(1 / \delta_{f}\right)-\left(i / 2 \omega_{f}\right)}\right)\right] .
$$

The constant $\varphi_{0}$ is $\varphi_{p}$ with $\delta_{S}=0$. Note that there is no directly-forced particular solution-the resonating basin modes dominate. ${ }^{6}$ This approximation can be used to give the approximate nonlinear interaction outside of the boundary current and recirculation region, resulting in

$$
\begin{aligned}
\delta_{I}^{2} \overline{J\left(\psi_{0}^{\prime}, \nabla^{2} \psi_{0}^{\prime}\right)}=\frac{2 m^{3} n \pi^{4} \omega_{f}^{4}\left|\varphi_{0}\right|^{2} \delta_{I}^{2}}{\delta_{S}^{2}} \sin (2 m \pi x) \sin (2 n \pi y) \\
\times\left(1-2(-1)^{m} e^{-\left(1 / \delta_{f}\right)} \cos \left(\frac{1}{2 \omega_{f}}\right)+e^{-\left(2 / \delta_{f}\right)}\right) .
\end{aligned}
$$

Expressing the interaction as a function of the average variance of the basin mode,

$$
\delta_{I}^{2} \overline{J\left(\psi_{0}^{\prime}, \nabla^{2} \psi_{0}^{\prime}\right)}=4 \pi^{4} m n\left(m^{2}+n^{2}\right) \delta_{I}^{2} \sin (2 m \pi x) \sin (2 n \pi y) \int_{0}^{1} \int_{0}^{1} \overline{\left(\psi_{0}^{\prime}\right)^{2}} \mathrm{dx} \mathrm{dy} .
$$

5. Often, a boundary-layer approximation such as $\overline{\psi_{0}} \approx(1-x) \sin (\pi y) e^{-x / \delta_{S}}+O\left(\delta_{S}\right)$ is presented as the solution to Stommel's model instead of the Stommel (1948) solution which is valid for all $\delta_{s}$. However, because the change in streamfunction in the boundary layer occurs in an $O\left(\delta_{S}\right)$ layer, the errors in the $x$-derivative are $O(1)$, and the approximation cannot be used to accurately estimate $J\left(\overline{\psi_{0}}, \nabla^{2} \overline{\psi_{0}}\right)$. For this reason, the solution valid for all $\delta_{S}$ is used here.

6. For this reason, the higher order in $\delta_{S}$ corrections do not strongly affect the interior solution. Thus, unlike the Stommel approximation, this approximation can be used to estimate $J\left(\overline{\psi_{0}}, \nabla^{2} \overline{\psi_{0}}\right)$. This was confirmed by calculating the nonlinear interaction of the full solution (with great effort by Mathematica). 
The mean vorticity flux convergence is very large within the western boundary current but $O\left(\delta_{S} \delta_{I}^{2}\right)$ outside it. On the other hand, the eddy term is $O\left(\left|\varphi_{0}\right|^{2} \delta_{I}^{2} / \delta_{S}^{2}\right)$ outside of the forcing region. ${ }^{7}$ Thus, while the eddy flux convergence is much smaller than the mean flux convergence in the boundary layer, it dominates outside of the boundary current. In fact, since the rate of change of vorticity in the boundary current instabilities is much larger than the wind input, $\varphi_{0}$ can be large enough for this term to be larger than the wind forcing in the interior as well. So if there is a resonating mode the first nonlinear correction to the interior solution is

$$
\frac{\partial \overline{\psi_{1}}}{\partial x}=-\delta_{I}^{2} \overline{J\left(\psi_{0}^{\prime}, \nabla^{2} \psi_{0}^{\prime}\right)}
$$

Combining the first two orders, a time-mean interior solution in a Sverdrup-like balance emerges, except that in addition to the wind forcing, the eddy vorticity flux convergence appears, to wit,

$$
\overline{\psi_{0}}+\overline{\psi_{1}}=(1-x) \sin (\pi y)+\int_{1}^{x} \delta_{I}^{2} \overline{J\left(\psi_{0}^{\prime}, \nabla^{2} \psi_{0}^{\prime}\right)} \mathrm{dx} .
$$

Thus, co-rotating and counter-rotating gyres will be superposed on the Sverdrup solution when the interior viscosity is small and the basin modes are resonated. These gyres can be viewed by subtracting the Sverdrup (1947) solution.

If more than one basin mode is resonated, a first-order contribution of their mutual interaction affects the time mean only if they are driven at the same frequency. In that case, resonance occurs when $1 / \omega_{f}=2 \pi \sqrt{n_{1}^{2}+m_{1}^{2}}=2 \pi \sqrt{n_{2}^{2}+m_{2}^{2}}$. For example, the square basin $(2,1)$ and $(1,2)$ mode interaction is

$$
\begin{aligned}
& \overline{J\left(\psi_{0}^{\prime}(1,2), \nabla^{2} \psi_{0}^{\prime}(2,1)\right)}+\overline{J\left(\psi_{0}^{\prime}(2,1), \nabla^{2} \psi_{0}^{\prime}(1,2)\right)} \\
& =\delta_{S}^{-2} 2 \pi^{4} \omega_{f}^{4}\left|\varphi_{0}(1,2) \| \varphi_{0}(2,1)\right|\left\{4 \pi \omega_{f} \cos (\pi x) \sin (\pi y) \sin (\phi)\right. \\
& \times[-2+6 \cos (2 \pi x)+14 \cos (2 \pi y)-18 \cos (2 \pi x) \cos (2 \pi y)] \\
& +\cos (\phi)(\sin (\pi x)-3 \sin (3 \pi x))(\sin (\pi y)-3 \sin (3 \pi y))\} .
\end{aligned}
$$

The constant $\phi$ is the phase difference between the modes. This cross-mode interaction is critical to the accuracy of the prediction in Figure 17. The phase constant was determined empirically for that figure.

This obvious flaw in this analytic model is that it does not predict which basin modes will be excited and what their amplitudes will be, as the periodic forcing used is just a proxy for the real instabilities. Nonetheless, by using the EOF diagnostics above, the

7. The terms involving $e^{-\left(1 / \delta_{f}\right)}$ represent the basin mode generated by the reflection of the directly-forced particular solution at the eastern boundary. These terms are negligible if $\delta_{f}$ is of the order of the recirculation gyre. Also, if the aspect ratio of the instability forcing is roughly one, then $m \omega_{f}$ and $n \omega_{f}$ are $O(1)$. 

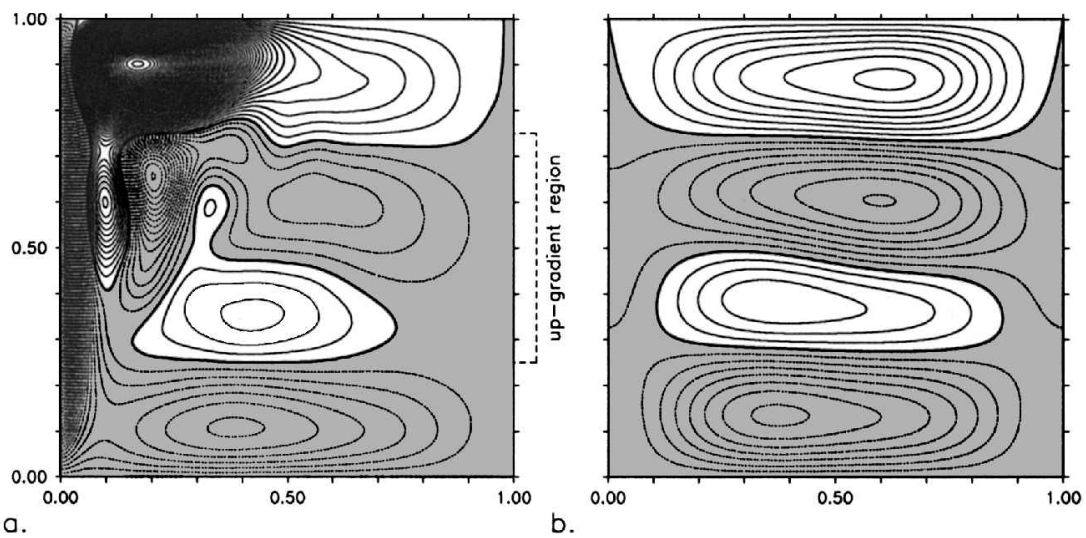

Figure 17. (a) The time-mean streamfunction minus the Sverdrup interior solution for the $\operatorname{Re}_{\mathrm{b}}=$ $0.25, \mathrm{Re}_{\mathrm{i}}=5$ calculation reveals a counter-rotating and co-rotating gyre pattern irreconcilable with a local down-gradient absolute vorticity flux. (b) The prediction using basin modes $(1,1)$, $(1,2),(2,1)$, and $(2,2)$ with variances from EOF diagnosis.

variance of each basin modes can be estimated. Setting the time-averaged, basin-averaged variance of an analytic basin mode equal to the sum of the squares of the singular values divided by the number of grid cells and time steps for the two EOFs for that basin mode gives the appropriate amplitude of each mode. The nonlinear interactions is determined using (22) and (26). For the calculation shown in Figure 15a, the five largest basin modes were used to predict the interior solution (25) was calculated using the analytic, linear form of the basin modes, i.e., (22) and (26). The resulting interior solution is shown in Figure $15 \mathrm{~b}$. There is a good agreement between the interior solution and the theory, and the dominant balance of terms in the interior is precisely as diagnosed from the numerical results.

Compare this wave-mean interaction with a down-gradient absolute vorticity flux. Because the absolute vorticity in the basin interior is dominated by the planetary vorticity background $\left(\delta_{I}^{2} \zeta+y \approx y\right.$ ), in order for the flux to be down-gradient the vorticity of the southern (northern) interior should be raised (lowered). The analysis above shows that the nonlinear interaction of the $n=1$ basin modes (which is proportional $\sin (2 \pi y)$ ) will also have this effect. However, this is coincidental, because if there is significant variability in modes where $n \neq 1$, then it becomes clear that the local absolute vorticity gradient is irrelevant to the shape of the resulting counter- and co-rotating gyres. Much of the variance in Figure 12 is in the second and third EOFs which are $n=2$ basin modes, and in this calculation counter-rotating and co-rotating gyres unrelated to the local absolute vorticity gradient occur (Fig. 17a). But, by diagnosing the basin modes present using the EOFs and calculating the resulting steady circulation, a similar interior solution-complete with 'up-gradient' regions-is produced.

The theory presented in this section is not complete. Primarily, it doesn't predict which 
basin modes will be excited. Perhaps an extension of Ben Jelloul and Huck (2003) to include the western boundary current region could close this model into a parameterization. Using the EOFs to generate the basin mode variance is an inexact method, and Figure $15 \mathrm{~b}$ could be improved by better estimates of the variances. Also, corrections of the next order, where the shape of each basin mode is affected by nonlinearity would improve the results. Obviously, including some of these effects would improve the agreement in Figures 15 and 17.

In summary, the co-rotating and counter-rotating gyres superposed on the Sverdrup (1947) interior solution are a natural result of resonating basin modes. The analysis reveals that although the first-order correction to the mean flow in the boundary current is the convergence of mean flux, in the interior the zero-frequency eddy flux convergence is the important nonlinear correction when the boundary current instabilities resonate the basin modes. This correction is responsible for the counter-rotating and co-rotating gyres and explains their nonlocal and 'up-gradient' characteristics. This theory relies on the weak nonlinearity of the basin interior and the resonance of basin modes. In a different parameter regime it is easy to imagine strong nonlinearities, such as coherent vortices leaking from the boundary current into the interior or exerting an important nonlocal effect (as suggested in Pasquero et al. (2001)), in which case the theory of Holm and Nadiga (2003) or Griffa and Castellari (1991) may be a more appropriate model. However, it seems unlikely that a Sverdrup balance will be possible in the presence of such strong eddy radiation from the boundary current. Thus, which limit is more representative of the ocean is an observational question. In the model used here, the nearly Sverdrup interior-in calculations with constant viscosity or controlled by boundary-enhanced viscosity — and the resonating basin modes provide the correct conditions for this theory.

\section{Summary and discussion}

Once large $\mathrm{Re}_{\mathrm{i}}$ has been reached, the single-gyre model exhibits many interesting behaviors. Through the use of boundary-enhanced viscosity representing unresolved physics, this limit can be studied in western-intensified calculations. In this paper, some of the interesting effects of the eddies that arise in the case of large $\mathrm{Re}_{\mathrm{i}}$ have been presented.

Once the interior viscosity is small enough, the nonlinear effects of eddies become pronounced. They play a critical role in the boundary layer, where an inviscid steady solution cannot exist. Radiation of waves from the strongly nonlinear boundary current plays a role in the interior, dominating the variability there. The nonlinear self-interaction of these waves produces steady counter-rotating and co-rotating gyres superposed on the Sverdrup flow. The temporal behavior is diverse, including a broad spectrum indicative of their weakly-damped nonlinear interaction and peaks at the natural frequencies of the basin. The slow adjustment of the strength and location of the recirculation gyre, linked to smaller eddies fluxing vorticity into these closed streamlines, leads to large variance, low frequency variability. With different parameters, the effects of eddies are able to exchange with the effects of friction, producing homoparic calculations. 
Demonstrating that the structure of the turbulent boundary layer proposed by Edwards and Pedlosky (1998) results in a western-intensified calculation with a larger inertial width than frictional width is an important addition to the extant boundary-layer theories. This extends the inviscid theory of Charney (1955) and its extension by Ierley and Ruehr (1986) to a boundary current structure capable of closing the basin vorticity budget. This boundary current structure features prominently in the multiple-gyre case as well (Fox-Kemper, 2004).

Although this model is highly idealized, it is likely that analogues of the inertial effects here will occur in more complex and inclusive models, with potentially significant implications. For example, typically ocean models run for climatological time scales are coarsely-resolved enough to be nearly non-inertial. However, the low-frequency motions found in this simple model (the adjustment of the recirculation gyre, nonlinear interactions, and the counter-rotating gyres) may offer climatologically significant intrinsic variability. The specific nature of this missing variability could have significant impacts on climate state transitions, as the variability of the boundary current or recirculation gyre may have a quite different effect from other variability. Thus, these results have some relevance in determining the type of variability to be externally imposed in coarse models to stimulate state transition.

Another example concerns the tuning of viscosity in ocean general circulation models. It is becoming common to have variable viscosity in these models (e.g., Large et al., 2001; Griffies and Hallberg, 2000). If homoparic equilibria exist in those models, the tuning must take into account not only the time-mean fields, but also the spatial distribution of the variability, to ensure that the tuning leads to the desired homoparic equilibrium. For example, if an inverse method (e.g., Wunsch, 1996) is used to determine the best viscosity for a model, then the cost function must include information about the variability as well as the mean state. Otherwise, the inverse method will fail to distinguish the multiple solutions available to minimize the errors in the mean state. If homoparic equilibria exist in other contexts, then physically-based closures, such as the local Smagorinsky (1963) nonlinear viscosity and the nonlocal 'alpha models' (e.g., Holm and Nadiga, 2003), should also be tested according to their predictions for variance (both resolved and implicit) as well as mean state.

Given the idea of vorticity flux exchange between friction and eddies presented in the companion paper, it is not surprising that the circulation strength can be controlled by the eddy fluxes. It is quite surprising, however, that the basin-scale features of the mean fields are so similar in homoparic calculations despite the profound changes to the boundary layer and eddies; there is no obvious constraint which requires that such a configuration must exist for each value of $\mathrm{Re}_{\mathrm{i}}$. Apparently, the Sverdrup interior and the shape of the recirculation gyre are tightly constrained, despite the mechanism of vorticity flux and the precise location of the boundary current relative to the boundary. Also, it is well-known that resolved eddies tend to flux vorticity strongly along the mean gradient rather than 
down it, and the frictional flux is, by definition, down-gradient. How it is that these fluxes end up producing such similar time means seems worthy of future inquiry.

The homoparic calculations themselves present an excellent tool for study of the compensating changes in friction and eddies. By following the contours in Figure $4 \mathrm{a}$, the changes in the eddies and friction can be studied without the complicating factor of significant changes to the mean flow. An excellent example might be to study the response the members of a homoparic pair to stochastic forcing. It has already been mentioned that the pair member with larger $\mathrm{Re}_{\mathrm{i}}$ took longer to spin up and had more variance about the mean. Thus, the two pair members may have significantly different responses to variable forcing as well.

In this sense, within a homoparic family, the spatially-variable viscosity acts as a parameterization of the eddies. More or less intense resolved eddies, and less or more 'eddy-viscosity fluxes,' can be selected. Furthermore, for simplicity, almost all of the calculations performed here were at the same resolution. However, the calculations with smaller $\mathrm{Re}_{\mathrm{i}}$ are unchanged by a significant reduction in resolution, so in this sense changing the viscosity to follow the homoparic contours allows for a 'parameterization' useful in coarse models. However, the friction variation along a homoparic contour is not a true parameterization, as there is no a priori knowledge about the location of the homoparic contours, nor any physical rationale for their location in parameter space.

The strong frequency dependence of the basin modes and subsequently the counterrotating gyres resulted from some of the instability frequencies of the boundary current being near to resonant frequencies of the basin. It is unclear whether basin modes will be resonated to the same degree in baroclinic models or models with realistic topography, or the ocean itself. Even the determination what the resonant modes of a basin with complex topography is non-trivial (Platzman, 1972; Primeau, 2002). However, the broadband spectrum of the more nonlinear calculations here suggests that for sufficiently high Reynolds number all frequencies will have some variance, including the resonant ones. Satellite observation of Rossby waves (e.g., Chelton and Schlax, 1996) is in its infancy, but already fairly large amounts of variance $(\approx 20 \%)$ in the ocean interior can be explained with Rossby and Kelvin waves (Fu et al., 1991; Webb and de Cuevas, 2003). Recent ship-based observations of the first baroclinic basin mode (Hagen, 2003) are also an encouraging confirmation of the relevance of this work. Correcting for the effects of mean shear on these waves may increase the variance explained (Killworth et al., 1997).

One might suggest that the presence of the basin modes might be inferred from the presence of counter-rotating gyres. However, the complications of local topography and local wind variation make this task nearly impossible. In principle, a steady numerical model could be used to produce a reference state with which to compare observations. However, in practice, the wind stress climatologies over the oceans are not known well enough even to produce consistent results across different wind products (Townsend et al., 2000). The bottom velocities are also poorly known, making the determination of the topographic stretching term in the linear vorticity equation difficult (Wunsch and 
Roemmich, 1985). The counter-rotating gyres are surely lost in the uncertainty of what the correct linear vorticity balance is. However, in analyzing the results of relatively inertial numerical models, they play an important role.

The counter-rotating gyres suggest that parameterizing the inertial effects of relatively inviscid, but weakly nonlinear, interior flows will be impossible with down-gradient mixing or even any local theory. If there are regions of the ocean driven by remotely-forced waves, must a high-resolution, eddy-permitting model of the boundary current be used to force them? Might a random variability model of the boundary current with the appropriate spectra suffice?

Stommel's simple single-gyre model still produces a number of interesting questions, many of which will have to be addressed in more realistic models in the future.

Acknowledgment. The author would like to thank his thesis advisors, J. Pedlosky and P. Malanotte-Rizzolifor their help and guidance. Thanks also to S. Jayne who contributed almost all of the computer resources. Discussions with G. Flierl, J. Franklin, M. Spall, V. Sheremet, R. Ferrari and $\mathrm{C}$. Wunsch greatly improved this work and its presentation. Useful comments were provided by two reviewers; one notable contribution was the idea for construction of Figure 4a. B.F-K. was supported in part by a ONR-supported NDSEG Fellowship, an MIT Presidential Fellowship, NSF OCE 9910654, a GFDL/Princeton University postdoctoral fellowship, and a NOAA Climate and Global Change postdoctoral fellowship (managed by UCAR).

\section{APPENDIX}

\section{Empirical Orthogonal Function Analysis}

This appendix describes the method of determination of the empirical orthogonal functions (EOFs) used here, and goes into detail about the representation of the basin modes by EOFs. The empirical orthogonal functions are discussed in detail elsewhere (for example, Berkooz et al. (1993) and Wunsch (1996)).

To generate the EOFs, the quantity of interest was interpolated onto a uniform $101 \times$ 101 grid at 10 time unit snapshots for a lengthy subinterval $(0(3000 / \beta L))$ of the integration time after equilibration. The EOFs were then calculated on this grid over this time interval, with uniform weighting to every grid point and time step.

Let $\mathbf{M}$ be the $\left(N_{x} N_{y}\right) \times N_{t}$ matrix of the field $\phi(x, y, t)$ interpolated onto the uniform grid at the snapshot times, such that a column of $\mathbf{M}$ contains all $\left(N_{x} N_{y}\right)$ of the grid points at a given time, and each row of $\mathbf{M}$ contains all of the $N_{t}$ snapshots of the value at a given location. Then the EOFs are the left singular vectors of $\mathbf{M}$ with their elements rearranged back onto the spatial grid.

$$
\mathbf{M}=\mathbf{U S V}^{T} .
$$

One of the matrices $\mathbf{U}$ and $\mathbf{V}$ is square of $\operatorname{size} \min \left(N_{x} N_{y}, N_{t}\right)^{2}$, and the other will be rectangular of size $\max \left(N_{x} N_{y}, N_{t}\right) \times \min \left(N_{x} N_{y}, N_{t}\right)$. Their columns are orthonormal (i.e., $\left.\mathbf{U U}^{T}=\mathbf{I}\right)$. The matrix $\mathbf{S}$ is square and nonzero only on the diagonal, with special 
values, the singular values, located on the diagonal. This singular vector decomposition can be performed on any matrix $\mathbf{M}$.

The heart of EOF analysis is the Eckart-Young-Mirsky theorem which states that the most efficient approximation (in a least-squares sense) to the matrix $\mathbf{M}$ with reduced degrees of freedom is

$$
\mathbf{M}_{i j} \approx \sum_{k=1}^{K} \mathbf{U}_{i k} \mathbf{S}_{k k} \mathbf{V}_{k j}^{T},
$$

where only the singular vectors (columns of $\mathbf{U}$ and $\mathbf{V}$ ) corresponding to the $K$ largest singular values are used in the sum (see, for example, Press et al., 1992). The MATLAB routine svds was used to calculate the first $\mathrm{K}$ singular vectors. Typically, calculating 20 singular vectors was sufficient to capture $>95 \%$ of the total variance, ${ }^{8}$ so that

$$
\sum_{i=1}^{N_{x} N_{y}} \sum_{j=1}^{N_{t}} \mathbf{M}_{i j}^{2} \approx \sum_{i=1}^{20} \mathbf{S}_{k k}^{2} .
$$

While only the first few EOFs are plotted in figures 9-13, the MATLAB calculation always involved determining more than 10 singular vectors.

The EOFs are the elements of the columns of $\mathbf{U}$ reordered back onto the spatial grid, or a truncated set of these singular vectors retaining only the ones with the largest singular values. Sometimes, the EOFs are defined instead as the orthogonal spatial basis functions which, when the variability is projected onto them, maximize the amount of covariance explained $\left(\operatorname{cov}\left(x_{i}, y_{j} ; x_{i^{\prime}}, y_{j^{\prime}}\right)=\sum_{\tau=1}^{N_{t}} \phi\left(x_{i}, y_{j}, t_{\tau}\right) \phi\left(x_{i^{\prime}}, y_{j^{\prime}}, t_{\tau}\right)\right)$. In this framework, the reduction of the basis is called the Karhunen-Loève decomposition. The covariance can also be produced as the $\left(N_{x} N_{y}\right) \times\left(N_{x} N_{y}\right)$ matrix, $\mathbf{M M}^{T}$. By multiplying (27) by its transpose, it is easy to see that the singular vectors will be the eigenvectors of this matrix, and the singular values will be the square root of the eigenvalues. Thus, the best truncated representation of the covariance will be formed from the eigenvectors with the largest eigenvalues, the same vectors which appear as columns of $\mathbf{U}$ in (28), the left singular vectors.

The columns of $\mathbf{V}$ in (27) are the right singular vectors and truncating them to a set retaining the maximum singular values maximize the amount of temporal covariance explained in a least-squares sense $\left(\operatorname{cov}\left(t_{\tau} ; t_{\tau^{\prime}}\right)=\sum_{i=1}^{N_{x}} \sum_{j=1}^{N_{y}} \Phi\left(x_{i}, y_{j}, t_{\tau}\right) \Phi\left(x_{i}, y_{j}, t_{\tau^{\prime}}\right)=\right.$ $\left.\mathbf{M}^{T} \mathbf{M}\right)$. They are used in this paper to indicate the projection of the field $\Phi$ onto the jth EOF over all time steps k:

$$
\sum_{i=1}^{N_{x} N_{y}} \mathbf{U}_{i k} \mathbf{M}_{i j}=\mathbf{S}_{j j} \mathbf{V}_{j k} .
$$

8. The exceptions were the determination of relative vorticity EOFs for the largest $\mathrm{Re}_{\mathrm{i}}$ calculations with boundary-enhanced viscosity, e.g., Figure 11 . The streamfunction variance was always easily captured. 
Thus, each column of $\mathbf{V}$ is the time series of the presence of each EOF.

The power spectrum density (PSD) of each column of $\mathbf{V}$ was estimated by the Thomson (1982) multi-taper method (time-bandwidth product $=4$, MATLAB routine $p m t m$ ) to provide frequency information for each EOF. The 95\% confidence intervals for the estimate plotted are those provided by the routine, which presume a chi-squared distribution. However, the numerical model is predictive rather than stochastic, so the 'noise' may not be distributed as expected, and the confidence interval should be considered a rough estimate.

So, a few EOFs with the largest singular values and their corresponding time series (the right singular vectors), give the most efficient representation of the full data series, as well as maximizing the amount of temporal and spatial covariance explained. Some care must be taken in interpreting the EOFs one by one, because they are empirical modes, rather than dynamical modes of the system.

For example, EOFs are roughly able to represent basin modes, but not exactly as one might desire. The free, linear basin modes have form and angular frequency given by:

$$
\begin{gathered}
\psi=\mathscr{R}\left[\psi_{0} \sin \left(m \pi x / x_{e}\right) \sin (n \pi y) e^{i\left[\omega_{m n} t+\left(x / 2 \omega_{m n}\right)\right]}\right], \\
\omega_{m n}=\frac{-1}{2 \pi \sqrt{m^{2} x_{e}^{-2}+n^{2}}} . \\
m, n=1,2,3, \ldots
\end{gathered}
$$

With trigonometric identities, (31) can be rewritten as a sum of two standing waves:

$$
\begin{aligned}
\psi & =\mathscr{R}\left(\psi_{0}\right) \sin \left(m \pi x / x_{e}\right) \sin (n \pi y)\left[\cos \left(\frac{x}{2 \omega_{m n}}\right) \cos \left(\omega_{m n} t\right)-\sin \left(\frac{x}{2 \omega_{m n}}\right) \sin \left(\omega_{m n} t\right)\right] \\
& -\Im\left(\psi_{0}\right) \sin \left(m \pi x / x_{e}\right) \sin (n \pi y)\left[\sin \left(\frac{x}{2 \omega_{m n}}\right) \cos \left(\omega_{m n} t\right)+\cos \left(\frac{x}{2 \omega_{m n}}\right) \sin \left(\omega_{m n} t\right)\right] .
\end{aligned}
$$

The real and imaginary part operators are $\mathscr{R}$ and $\Im$. The spatial patterns of the standing waves (in brackets) do not change as the phase of the wave changes, only their magnitude changes with time. Because there are two standing waves required to represent the total propagating wave, for a single basin mode there will be exactly two EOFs.

EOFs are required to be spatially orthogonal by definition, yet the basin mode standing waves are not orthogonal in $x$ with uniform weighting on a uniform grid. Expressing the matrices and vectors as functions evaluated at the grid points and time steps,

$$
M\left(x_{i}, y_{j}, t_{\tau}\right) \approx \sum_{k=1}^{K} u_{k}\left(x_{i}, y_{j}\right) s_{k} v_{k}\left(t_{\tau}\right)
$$

The basin modes are already orthogonal in time, up to indeterminacy of degenerate frequencies. Every orthonormal basis will be of the form (for some $\delta$ ) 


$$
\begin{aligned}
& v_{1}\left(t_{\tau}\right)=\frac{\cos \left(\omega_{m n} t_{\tau}+\delta\right)}{\left|\cos \left(\omega_{m n} t_{\tau}\right)\right|} \approx \frac{\cos \left(\omega_{m n} t_{\tau}+\delta\right)}{\sqrt{N_{t} / 2}}, \\
& v_{2}\left(t_{\tau}\right)=\frac{\sin \left(\omega_{m n} t_{\tau}+\delta\right)}{\left|\sin \left(\omega_{m n} t_{\tau}\right)\right|} \approx \frac{\sin \left(\omega_{m n} t_{\tau}+\delta\right)}{\sqrt{N_{t} / 2}} .
\end{aligned}
$$

The root of the inner product over the domain of a function is notated with bar brackets, e.g., $|\Theta(t)| \equiv \sqrt{\sum_{\tau=1}^{N_{t}}\left(\Theta\left(t_{\tau}\right)\right)^{2}}$ and $|\Xi(x)| \equiv \sqrt{\sum_{i=1}^{N_{x}}\left(\Xi\left(x_{i}\right)\right)^{2}}$. The approximation is valid in the limit that the sampling time $\Delta t$ and total analysis interval obey $N_{t} \Delta t \gg 1 / \omega_{m n} \gg \Delta t$, a condition easily satisfied.

The basin modes are already orthogonal in $y$, so just as in time, the dependence of the singular vectors on $y$ naturally becomes a discrete Fourier transform. For the two standing waves of a single basin mode there is only one function in $y$ to account for, $\sin (n \pi y)$. Using the function $u_{k}$ which determines the values in the kth column of $\mathbf{U}$,

$$
\begin{aligned}
& u_{1}\left(x_{i}, y_{j}\right)=\frac{f_{1}\left(x_{i}\right) \sin \left(n \pi y_{j}\right)}{\left|\sin \left(n \pi y_{j}\right)\right|} \approx \frac{f_{1}\left(x_{i}\right) \sin \left(n \pi y_{j}\right)}{\sqrt{N_{y} / 2}}, \\
& u_{2}\left(x_{i}, y_{j}\right)=\frac{f_{2}\left(x_{i}\right) \sin \left(n \pi y_{j}\right)}{\left|\sin \left(n \pi y_{j}\right)\right|} \approx \frac{f_{2}\left(x_{i}\right) \sin \left(n \pi y_{j}\right)}{\sqrt{N_{y} / 2}} .
\end{aligned}
$$

The approximation is valid whenever the meridional wavelength is much longer than the grid spacing.

The complication lies in the $x$-dependence. Orthonormalization of the basin modes provides a basis spanning all sums of the two standing wave functions in $x$. For a two-dimensional space, all of the orthonormal bases differ by a single rotation. First, denote the standing wave $x$-dependence by

$$
\begin{gathered}
\Phi_{1}\left(x_{i}\right) \equiv \mathscr{R}\left(\psi_{0}\right) \sin \left(m \pi x / x_{e}\right) \cos \left(\frac{x}{2 \omega_{m n}}\right)-\Im\left(\psi_{0}\right) \sin \left(m \pi x / x_{e}\right) \sin \left(\frac{x}{2 \omega_{m n}}\right), \\
\Phi_{2}\left(x_{i}\right) \equiv-\mathscr{R}\left(\psi_{0}\right) \sin \left(m \pi x / x_{e}\right) \sin \left(\frac{x}{2 \omega_{m n}}\right)-\Im\left(\psi_{0}\right) \sin \left(m \pi x / x_{e}\right) \cos \left(\frac{x}{2 \omega_{m n}}\right),
\end{gathered}
$$

so that

$$
\psi\left(x_{i}, y_{j}, t_{\tau}\right)=\sin \left(n \pi y_{j}\right)\left[\Phi_{1}\left(x_{i}\right) \cos \left(\omega_{m n} t_{\tau}\right)+\Phi_{2}\left(x_{i}\right) \sin \left(\omega_{m n} t\right)\right] .
$$

Consider the following combination which gives all possible orthonormalizations (for some $\alpha$ ),

$$
\begin{aligned}
& f_{1}\left(x_{i}\right)=\frac{\phi_{1}\left(x_{i}\right)+\phi_{2}\left(x_{i}\right)}{\left|\phi_{1}+\phi_{2}\right|} \sin (\alpha)+\frac{\phi_{2}\left(x_{i}\right)-\phi_{1}\left(x_{i}\right)}{\left|\phi_{2}-\phi_{1}\right|} \cos (\alpha), \\
& f_{2}\left(x_{i}\right)=\frac{\phi_{1}\left(x_{i}\right)+\phi_{2}\left(x_{i}\right)}{\left|\phi_{2}+\phi_{1}\right|} \cos (\alpha)-\frac{\phi_{2}\left(x_{i}\right)-\phi_{1}\left(x_{i}\right)}{\left|\phi_{2}-\phi_{1}\right|} \sin (\alpha) .
\end{aligned}
$$


The functions $\phi \equiv \Phi /|\Phi|$ are the normalized standing waves. The reader can verify that this combination is normal and orthogonal. By inverting (42), the standing waves can be expressed as linear combinations of these orthogonal functions. So, the projections of the basin modes onto the orthogonal basis functions are

$$
\begin{aligned}
& \sum_{\forall: x} \Phi_{1}(x) f_{1}(x)=\frac{\left|\Phi_{1}\right|}{2}\left[-\left|\phi_{2}-\phi_{1}\right| \cos (\alpha)+\left|\phi_{1}+\phi_{2}\right| \sin (\alpha)\right], \\
& \sum_{\forall: x} \Phi_{1}(x) f_{2}(x)=\frac{\left|\Phi_{1}\right|}{2}\left[\left|\phi_{1}+\phi_{2}\right| \cos (\alpha)+\left|\phi_{2}-\phi_{1}\right| \sin (\alpha)\right], \\
& \sum_{\forall: x} \Phi_{2}(x) f_{1}(x)=\frac{\left|\Phi_{2}\right|}{2}\left[\left|\phi_{2}-\phi_{1}\right| \cos (\alpha)+\left|\phi_{1}+\phi_{2}\right| \sin (\alpha)\right], \\
& \sum_{\forall: x} \Phi_{2}(x) f_{2}(x)=\frac{\left|\Phi_{2}\right|}{2}\left[\left|\phi_{1}+\phi_{2}\right| \cos (\alpha)-\left|\phi_{2}-\phi_{1}\right| \sin (\alpha)\right] .
\end{aligned}
$$

For a full bi-orthogonalization as required by the singular value decomposition, we must now adjust $\alpha$ and $\delta$ to produce a choice of $\delta$ which leaves the matrix $\mathbf{S}$ diagonal. It is easily demonstrated using (37), (41), and (43) that

$$
\begin{aligned}
\frac{4 \sum_{\forall: x, y, t} u_{1}(x, y) M(x, y, t) v_{1}(t)}{\sqrt{N_{y} N_{t}}}= & \left|\phi_{1}+\phi_{2}\right| \sin (\alpha)\left[\left|\Phi_{1}\right| \cos (\delta)-\left|\Phi_{2}\right| \sin (\delta)\right]+ \\
& \left|\phi_{2}-\phi_{1}\right| \cos (\alpha)\left[-\left|\Phi_{1}\right| \cos (\delta)-\left|\Phi_{2}\right| \sin (\delta)\right],
\end{aligned}
$$

$$
\begin{aligned}
\frac{4 \sum_{\forall: x, y, t} u_{1}(x, y) M(x, y, t) v_{2}(t)}{\sqrt{N_{y} N_{t}}}= & \left|\phi_{1}+\phi_{2}\right| \sin (\alpha)\left[\left|\Phi_{1}\right| \sin (\delta)+\left|\Phi_{2}\right| \cos (\delta)\right]+ \\
& \left|\phi_{2}-\phi_{1}\right| \cos (\alpha)\left[-\left|\Phi_{1}\right| \sin (\delta)+\left|\Phi_{2}\right| \cos (\delta)\right],
\end{aligned}
$$

$$
\begin{array}{r}
\frac{4 \sum_{\forall: x, y, t} u_{2}(x, y) M(x, y, t) v_{1}(t)}{\sqrt{N_{y} N_{t}}}=\left|\phi_{1}+\phi_{2}\right| \cos (\alpha)\left[\left|\Phi_{1}\right| \cos (\delta)-\left|\Phi_{2}\right| \sin (\delta)\right]+ \\
\left|\phi_{2}-\phi_{1}\right| \sin (\alpha)\left[\left|\Phi_{1}\right| \cos (\delta)+\left|\Phi_{2}\right| \sin (\delta)\right],
\end{array}
$$

$$
\begin{array}{r}
\frac{4 \sum_{\forall: x, y, t} u_{2}(x, y) M(x, y, t) v_{2}(t)}{\sqrt{N_{y} N_{t}}}=\left|\phi_{1}+\phi_{2}\right| \cos (\alpha)\left[\left|\Phi_{1}\right| \sin (\delta)+\left|\Phi_{2}\right| \cos (\delta)\right]+ \\
\left|\phi_{2}-\phi_{1}\right| \sin (\alpha)\left[\left|\Phi_{1}\right| \sin (\delta)-\left|\Phi_{2}\right| \cos (\delta)\right] .
\end{array}
$$

Setting (45) and (46) to zero gives simultaneous equations on $\alpha$ and $\delta$ to make the matrix $\mathbf{S}$ diagonal. The remaining equations (44) and (47) produce the two non-zero singular values. As these two EOFs will exactly reproduce the basin mode, there will only be two nonzero singular values. 
The superposition of multiple basin modes is not so simple. In the preceding calculation all linear combinations of the sine and cosine of the same frequency just resulted in amplitude and phase changes, so it was easy to determine the temporal singular vectors. Likewise, once an orthonormal basis was found to span the space of the standing waves, then a simple rotation produced all of the possible orthonormal bases. In general, the linear combination of multi-frequency sines and cosines would not be easy. For the linear basin modes, when two modes with the same $y$ wavenumber but different $x$ wavenumber are superposed, they will have different frequencies, but have non-zero projections onto one another. A biorthogonalization of these modes in $x$ and time would again be required, but the four resulting singular vectors would each possess some of the variance of each of the two basin modes.

However, simple experiments combining a number of linear basin modes and processing them as the numerical model output was processed revealed many facts. (1) Low-wavenumber basin modes were reproduced accurately in space. (2) The EOFs occurred with the correct frequency with two EOFs per basin mode. (3) The EOFs resembled the standing waves of the basin modes with an arbitrary phase difference. (4) When the $y$-wavenumber of the basin modes was the same, some separation remained so that each EOF still possessed a dominant frequency, but the modes were mixed. By choosing a different weighting function, the basin modes could be made orthogonal, but in that case the resulting EOFs would be biased toward detecting basin modes and would no longer minimize the uniformlyaveraged covariance.

When the basin modes occur on a mean flow, they will be affected by it and no longer be orthogonal in $y$ or $x$. In this case, distinguishing between the $(1,2)$ and the $(2,1)$ basin mode becomes less meaningful, as any basis spanning the spatial pattern of oscillations at that frequency will do. The projection of a non-degenerate basin mode onto the EOFs may be dominated by two EOFs, but it will have a nonzero projection onto some others; the linear basin modes are not orthogonal in $x$, and the nonlinear basin modes are not orthogonal in $y$. All of the EOFs shown in Figure 9, for example, have some signal in the PSD at all of the frequencies present in the PSDs for other EOFs. However, note that the largest peak in the PSD is typically many orders of magnitude larger than the next largest, allowing reasonable confidence in the association of these modes with the frequencies of their largest peak. This remains true even for the highest Reynolds number calculations studied.

The EOF analysis is biased toward detecting phenomena which are fixed in space and temporally reoccurring, so in addition to the mixing of basin mode signals, other effects are almost certainly aliased into the EOF signal. Other approaches have been suggested to better quantify propagating modes (Barnett, 1983), but this EOF analysis seems adequate for the purposes of this study. 


\section{REFERENCES}

Barcilon, V. 1998. On the barotropic ocean with bottom friction. J. Mar. Res., 56, 731-771.

Barnett, T. P. 1983. Interaction of the monsoon and Pacific trade wind systems at interannual time scales. Part I: The equatorial zone. Mon. Weather Rev., 111, 756-773.

Ben Jelloul, M. and T. Huck. 2003. Basin-mode interactions and selection by the mean flow in a reduced-gravity quasigeostrophic model. J. Phys. Oceanogr., 33, 2320-2332.

Berkooz, G., P. Holmes and J. L. Lumley. 1993. The proper orthogonal decompositionin the analysis of turbulent flows. Ann. Rev. Fluid Mech., 25, 539-575.

Charney, J. G. 1955. The Gulf Stream as an inertial boundary layer. Proc. Nat. Acad. Sci., 41, 731-740.

Chelton, D. B. and M. G. Schlax. 1996. Global observations of oceanic Rossby waves. Science, 272, 234-238.

Edwards, C. A. and J. Pedlosky. 1998. Dynamics of nonlinear cross-equatorialflow. Part I: Potential vorticity transformation. J. Phys. Oceanogr., 28, 2382-2406.

Fofonoff, N. P. 1954. Steady flow in a frictionless homogenous ocean. J. Mar. Res., 13, $254-262$.

Fox-Kemper, B. 2003. Friction and Eddies: Removal of Vorticity from the Wind-Driven Gyre. Ph.D. Thesis. MIT/WHOI, 2003-06,310 pp.

2004. Re-evaluating the roles of eddies in multiple barotropic wind-driven gyres. J. Phys. Oceanogr., (submitted).

Fox-Kemper, B. and J. Pedlosky. 2004. Wind-driven barotropic gyre I: Circulation control by eddy vorticity fluxes to a region of enhanced removal. J. Mar. Res., 62, 169-193.

Fu, L. L., J. Vazquez and C. Perigaud. 1991. Fitting dynamic models to the GEOSAT sea level observations in the tropical Pacific Ocean, Part I: A free wave model. J. Phys. Oceanogr., 21, $798-809$.

Greatbatch, R. J. and B. T. Nadiga. 1999. Four-gyre circulation in a barotropic model with double-gyre wind forcing. J. Phys. Oceanogr., 30, 1461-1471.

Greenspan, H. P. 1962. A criterion for the existence of inertial boundary layers in the oceanic circulation. Proc. Nat. Acad. Sci., 48, 2034-2039.

Griffa, A. and S. Castellari. 1991. Nonlinear general circulation of an ocean model driven by wind with a stochastic component. J. Mar. Res., 49, 53-73.

Griffa, A. and R. Salmon. 1989. Wind-driven ocean circulation and equilibrium statistical mechanics. J. Mar. Res., 47, 457-492.

Griffies, S. M. and R. W. Hallberg. 2000. Biharmonic friction with a Smagorinsky-like viscosity for use in large-scale eddy-permitting ocean models. Mon. Weath. Rev., 128, 2935-2946.

Hagen, E. 2003. Baroclinic Rossby waves in the northeast Atlantic Ocean? Beiträge zur Klima- und Meeresforschung, Aus Anlass des 70. Geburtstages von Peter Hupfer, F. M. Chmielewski and T. Foken, eds., Selbstverlag, Berlin, Bayreuth, 149-158.

Holland, W. R. and L. B. Lin. 1975. On the origin of mesoscale eddies and their contribution to the general circulation of the ocean. II. A parameter study. J. Phys. Oceanogr., 5, 658-669.

Holm, D. D. and B. T. Nadiga. 2003. Modeling mesoscale turbulence in the barotropic double gyre circulation. J. Phys. Oceanogr., 33, 2355-2365.

Ierley, G. R. and O. G. Ruehr. 1986. Analytic and numerical solutions of a nonlinear boundary-layer problem. Studies in Appl. Math, 75, 1-36.

Ierley, G. R. and V. A. Sheremet. 1995. Multiple solutions and advection-dominated flows in the wind-driven circulation. Part I: Slip. J. Mar. Res., 53, 703-737.

Il'in, A. M. and V. M. Kamenkovich. 1964. The structure of the boundary layer in a two-dimensional model of ocean currents. Okeanologiya, 4, 756-769, in Russian.

Kamenkovich, V. M., V. A. Sheremet, A. R. Pashtushkov and S. Belotserkovsky. 1995. Analysis of 
the barotropic model of the subtropical gyre in the ocean for finite Reynolds numbers, Part I. J. Mar. Res., 53, 959-994.

Killworth, P. D., D. B. Chelton and R. A. de Szoeke. 1997. The speed of observed and theoretical long extratropical planetary waves. J. Phys. Oceanogr., 27, 1946-1966.

Large, W. G., G. Danabasoglu, J. C. McWilliams, P. R. Gent and F. O. Bryan. 2001. Equatorial circulation of a global ocean climate model with anisotropic horizontal viscosity. J. Phys. Oceanogr., 31, 518-536.

Meacham, S. P. 2000. Low-frequency variability in the wind-driven circulation. J. Phys. Oceanogr., 30, 269-293.

Moore, D. W. 1970. The mass transport velocity induced by free oscillations at a single frequency. Geophys. Fluid Dyn., 1, 237-247.

Munk, W. H. 1950. On the wind-driven ocean circulation. J. Meteor., 7, 79-93.

Özgökmen, T. and E. P. Chassignet. 1998. Emergence of inertial gyres in a two-layer quasigeostrophic ocean model. J. Phys. Oceanogr., 28, 461-484.

Pasquero, C., A. Provenzale and A. Babiano. 2001. Parameterization of dispersion in twodimensional turbulence. J. Fluid Mech., 439, 279-303.

Pedlosky, J. 1965a. A note on the western intensification of the oceanic circulation. J. Mar. Res., 23, 207-209.

- 1965b. A study of the time dependent ocean circulation. J. Atmos. Sci., 22, 267-272.

Platzman, G. W. 1972. Two-dimensional free oscillations in natural basins. J. Phys. Oceanogr., 2, 117-137.

Press, W. H., S. A. Teukolsky, W. T. Vetterling and B. P. Flannery. 1992. Numerical Recipes, Cambridge University Press, Cambridge, 2nd ed., 993 pp.

Primeau, F. 2002. Long Rossby wave basin-crossing time and the resonance of low-frequency basin modes. J. Phys. Oceanogr., 32, 2652-2665.

Salmon, R., G. Holloway and M. C. Hendershott. 1976. The equilibrium statistical mechanics of simple quasi-geostrophicmodels. J. Fluid Mech., 75, 691-703.

Sheremet, V. A., G. R. Ierley and V. M. Kamenkovich. 1997. Eigenanalysis of the two-dimensional wind-driven ocean circulation problem. J. Mar. Res., 55, 57-92.

Sheremet, V. A., V. M. Kamenkovich and A. R. Pashtushkov. 1995. Analysis for the barotropic model of the subtropical gyre in the ocean for finite Reynolds numbers, Part II. J. Mar. Res., 53, 995-1024.

Smagorinsky, J. 1963. General circulation experiments with the primitive equations I: The basic experiment. Mon. Weath Rev., 91, 99-164.

Stommel, H. M. 1948. The westward intensification of wind-driven ocean currents. Trans. Amer. Geophys. Union, 29, 202-206.

Sverdrup, H. U. 1947. Wind-driven currents in a baroclinic ocean; with application to the equatorial currents of the eastern Pacific. Proc. Nat. Acad. Sci., 33, 318-326.

Thomson, D. J. 1982. Spectral estimation and harmonic analysis. Proc. IEEE, 70, 1055-1092.

Townsend, T. L., H. E. Hurlburt and P. J. Hogan. 2000. Modeled Sverdrup flow in the North Atlantic from 11 different wind stress climatologies. Dyn. Atmos. Oceans, 32, 373-417.

Webb, D. J. and B. A. de Cuevas. 2003. The region of large sea surface height variability in the Southeast Pacific Ocean. J. Phys. Oceanogr., 33, 1044-1056.

Wunsch, C. 1996. The Ocean Circulation Inverse Problem, Cambridge University Press, Cambridge, $442 \mathrm{pp}$.

Wunsch, C. and D. Roemmich. 1985. Is the North Atlantic in Sverdrup balance? J. Phys. Oceanogr., $15,1876-1880$. 